\title{
Cavity Resonance Suppression Using Fluidic Spoilers
}

\author{
Gareth J. Bennett,_* Patrick N. Okolo,£ and Kun Zhao \\ Trinity College Dublin, University of Dublin, Dublin D02 PN40, Ireland \\ and \\ John Philo, \pm Yaoyi Guan, $\underline{\S}$ and Scott C. Morris $\mathbb{1}$ \\ University of Notre Dame, Notre Dame, Indiana 46556
}

DOI: $\underline{10.2514 / 1 . J 057407}$

\begin{abstract}
This paper examines experimentally the use of a fluidic spoiler to suppress the resonance within a partially closed cylindrical cavity subject to a grazing flow. The relative movement of aircraft and high-speed land-based vehicles through air often results in structural cavities in these vehicles being subject to shear-layer-driven resonance. This can lead to high-amplitude pressure fluctuations within the cavity volume, causing damage to stores or equipment found within landing-gear wheel or weapon bays, for example, or else significant discomfort to the passengers of cars or trains. This large-scale buffeting can also cause vehicle stability problems and can increase drag. This work presents a novel method, in which passive flow control consisting of an upstream fluidic spoiler is used to redirect the upstream flow so that the cavity orifice is shielded. As a result, the grazing flow can no longer detach from the upstream leading edge of the cavity, and thus, vortex shedding is suppressed. The scope of the study includes an examination of higherorder azimuthal acoustic modes excited in the cylindrical cavity: modes which have received little attention in the literature, but which can be readily excited for many flow configurations for partially covered cavities.
\end{abstract}

\section{Nomenclature}

$A=$ area of orifice opening, $\mathrm{m}^{2}$

$b_{1 / 2}=$ fluidic-spoiler half-width, $\mathrm{m}$

$c=$ speed of sound, $\mathrm{m} / \mathrm{s}$

$c_{b}=$ spreading-rate proportionality constant

$c_{j}=$ centerline-trajectory proportionality constant

$D=$ diameter of the cylindrical cavity, $\mathrm{m}$

$f_{\mathrm{HR}}=$ Helmholtz resonance frequency; $c / 2 \pi \sqrt{A / V l_{s}}, \mathrm{~Hz}$

$H=$ height/depth of the cylindrical cavity, $\mathrm{m}$

$H e=$ Helmholtz number; $2 \pi f R / c$

$L=$ streamwise length of orifice opening, $\mathrm{m}^{2}$

$l_{m}=$ momentum length scale, $\mathrm{m}$

$l_{s}=$ length of slug of air in neck of Helmholtz resonator, $\mathrm{m}$

$M \quad=$ Mach number

$M_{o}=$ initial kinematic momentum flux, $\mathrm{m}^{3} / \mathrm{s}^{2}$

$n=$ order of the shear-layer mode

$R=$ velocity ratio; $U_{\text {jet }} / U_{\infty}$

$R_{c}=$ radius of the cylindrical cavity, $\mathrm{m}$

$S r=$ Strouhal number; $f L / U \approx(n-\alpha) /(M+(1 / \kappa))$

$U_{\text {jet }}=$ planar-jet velocity at slot exit, $\mathrm{m} / \mathrm{s}$

$U_{\infty}=$ freestream crossflow velocity, $\mathrm{m} / \mathrm{s}$

$V=$ volume of cavity, $\mathrm{m}^{3}$

$W \quad=\quad$ width of square wind-tunnel test section, $\mathrm{m}$

$w \quad=\quad$ width of the planar-jet slot in the streamwise direction, $\mathrm{m}$

$x, y=$ streamwise, vertical coordinates, $\mathrm{m}$

$\alpha=$ phase delay

$\Delta=$ measure of orifice offset from downstream edge of cylindrical cavity, $\mathrm{m}$ $\begin{aligned} \kappa \quad= & \text { convection velocity of the shear layer normalized by the } \\ & \text { freestream velocity } \\ \omega \quad & \text { circular frequency, } \mathrm{rad} / \mathrm{s}\end{aligned}$

\section{Introduction}

$\mathbf{L}$ OW-MACH-NUMBER cavity flows exist in numerous engineering applications. These systems are often susceptible to resonance based on feedback between the internal cavity pressure and the shear layer (SL) over the cavity opening. This phenomenon is important to understand because the unsteady pressure fluctuations may be very large, and can result in undesirable noise or damage to the cavity structure or equipment inside the cavity. These flows have been studied by a large number of researchers, including a few mentioned here to illustrate the wide range of applications. A study by Langtry and Spalart [1] used computational methods to predict the unsteady pressure inside a landing-gear wheel well on commercial aircraft geometry. Balasubramanian et al. [2] considered "sunroof buffeting" on simplified vehicle geometry. Nakiboglu et al. [3] studied a series of side branch resonators as a model for flow in a corrugated pipe. Aly and Ziada [4] examined higher-order modes in a pipe system with a sudden diameter change, and investigated the effect of cavity depth on acoustic particle velocity.

In an attempt to suppress cavity noise, a number of different methods have been investigated: active fluid-injection methods, such as microjets $[\underline{5}, 6]$ and leading-edge blowing [7]; plasma actuators $[8,9]$; the installation of a crossflow rod at the cavity leading edge $[10,11] ;$ and the installation of Helmholtz resonators within the cavity environment [12]. There have been a number of excellent reviews of cavity noise dynamics and suppression published to date [13,14].

SL-driven cavity flows can exhibit several types of features generally described as resonance. The review paper of Rockwell and Naudascher [15] categorized self-sustaining oscillations into three groups: fluid dynamic, fluid resonant, and fluid elastic. Within the fluid-dynamic category, a highly cited work is for high-Machnumber $(M>0.5)$ flow over a shallow cavity known as a Rossiter cavity [16]. In this system, the feedback mechanism is an upstreamtraveling acoustic wave generated by turbulent structures impacting the downstream edge of the cavity. These acoustic waves have a wavelength of the same order of magnitude as the opening length. Resonance occurs if this acoustic frequency excites the SL oscillation. This type of feedback mechanism is not expected to lead to cavity resonance in the current system.

In this paper, the self-sustaining cavity oscillations, which are strongly coupled with resonant waves within the SL and which are 
classified as fluid-resonant oscillations, are of interest. For this type, SL cavity flows can excite standing acoustic waves in the crossstream direction, particularly when this spanwise dimension, $H$, being the height of the cavity, is large when compared to the streamwise dimension, $L$. These modes are often called longitudinal or depth modes. For these modes, the acoustic waves are reflected from the bottom of the cavity and can feedback to the SL. These standing waves reflect from the end of the cavity and any of 1) the top of a cavity that is closed except for a small opening; 2) the opposite side of the wind tunnel, if the experiment is performed in a closed section; 3) between the ends of two opposing cavities in the case of a "coaxial" resonator configuration; or 4) between the bottom of the cavity and the change of impedance due to the opening. Depth modes for deep cavities were studied by Yang et al. [17], who specifically analyzed the effect of the streamwise dimension of the cavity. A coaxial side branch configuration was studied by Oshkai and Yan [18].

To date, within the fluid-resonant category, little consideration has been given to higher-order acoustic modes that might resonate in cavities, whether cylindrical or polyhedral in shape. Bennett et al. [19] examined their excitation through a particle image velocimetry (PIV) study of the SL over the orifice, Verdugo et al. [20] identified the noise source and sink in the SL using an experimental method employing Howe's analogy, and Stephens et al. [21] studied the effect of orifice location and characteristics on the mode excited. In aeroacoustics, a study by Marsden et al. [22] was performed examining cylindrical burst-disk cavities and vent holes located under wings, which they stated have been subject to little investigation, despite being clearly identifiable in flyover measurements. In this current paper, we are particularly interested in exploring test cases, in which both planewave longitudinal modes and also azimuthal modes are excited. The azimuthal modes can, in turn, be coupled with the plane-wave modes depending on flow speed or orifice geometry.

In addition to standing acoustic waves, an SL-driven cavity can act also as an Helmholtz resonator (HR), in which the oscillation is caused instead by compression of the volume of fluid inside the cavity. This is a third mode type of interest in the current paper. The preferred resonance frequency of this kind of system can be calculated as

$$
f_{\mathrm{HR}}=\frac{c}{2 \pi} \sqrt{\frac{A}{V l_{s}}}
$$

in which $A$ is the area of the cavity opening; $V$ is its volume; and, in this work, $l_{s}$ is the length of the slug of air that oscillates in the opening, or neck, of the cavity. For the cavity under consideration in this work, the neck length is essentially zero. However, the air in the internal and external regions of the neck tends to move in unison, and so, similar to the development of equivalent length corrections for open-ended ducts, this behavior has resulted in the investigation of an "effective" neck length in the literature. In this study, $l_{s}$ is chosen to be $L$, the streamwise length of the orifice, and was found to match the experimental data well. This length has been used by both Kook and Mongeau [23] and Ma et al. [24] who have both studied HRs and accurately predicted the magnitude of the interior cavity pressure when adequate information about the SL is available.

The air curtain has been used in a number of diverse engineering applications [25-28]. In aeronautics, it was first proposed for use for landing-gear noise reduction by Wickerhoff and Sijpkes [29], after which Oerlemans and de Bruin [30] performed proof-of-concept research to validate it. The planar jet as a means to reduce noise has also been investigated by Zhao et al. [31,32], in which tandem rods were examined as a simplified representation of aircraft landing gear. Subsequently, the dual air curtain has been developed and investigated, in which improved acoustic and energy efficiency have been achieved through the addition of a second upstream planar jet $[33,34]$.

This paper examines experimentally the use of a fluidic spoiler to suppress the acoustic resonance within a partially closed cylindrical cavity subject to a grazing flow. The work presents a novel method of passive flow control, which consists of an upstream fluidic spoiler, which might also be called an air curtain or simply an upstream planar jet in crossflow. The fluidic spoiler is used to redirect the upstream flow so that the cavity orifice is shielded. As a result, the grazing flow can no longer detach from the upstream leading edge of the cavity, and thus, vortex shedding is suppressed.

Current/ongoing research in the United States involves the testing of thin airflow streams as a means to reduce noise: 1) the high-flow bypass concept of the Rolls-Royce Tay turbofan engine for the Gulfstream X-54 demonstrator, which routes captured air in a smooth flowpath around the power plant to enclose it in an aerodynamically shaped aeroshell; and 2) jet noise reduction in the novel GE engine Fluid Shield technology within the flagship activity of the Pentagon's Versatile Affordable Advanced Turbine Engines research program. The current paper sits in this nascent research field of redirecting flow to reduce noise/pressure fluctuations.

\section{Fluid Mechanics of a Planar Jet in Crossflow}

\section{A. Theoretical Model}

Unlike the circular jet issuing into quiescent air, which has important engineering applications related to aeroengine design and its associated environmental noise, planar jets have attracted far less research interest. Their main application has been in the blockage of tunnels and corridors to prevent smoke and pollutant transmission, and for doorways to prevent heat loss. These applications are characterized by the fact that they are a full-span virtual screen for the passageway or doorway, which creates a pressure drop to inhibit heat and mass transfer between the adjoining fluids yet with the benefit of allowing the transit of people and vehicles. The planar jet in a crossflow, which, rather than creating a blockage, instead, redirects flow to shield a solid body, is a much more novel application and one that has appeared in the literature recently, and, in this paper, is referred to as a fluidic spoiler. For such applications, in which the planar jet does not impinge on an opposing surface, such as in heat transfer applications, for example, a study by Ramaprian and Haniu [35] provides a simple semi-empirical model to predict the trajectory of such flows. Applications foreseen by these authors were cooling tower plumes, smokestack exhausts, thermal discharges into rivers, etc., and in their work, they examined two-dimensional (2-D) buoyant (heated) and nonbuoyant jets issuing into a hydraulic flume perpendicular to the direction of the flow. To describe the behavior of a 2-D jet discharged vertically with a velocity of $U_{\text {jet }}$ into a horizontal crossflow of velocity $U_{\infty}$, the authors performed a dimensionless analysis of the relevant variables, and found that the trajectory of maximum velocity along the centerline of the jet scales with a length scale based on the exit kinematic momentum flux and follows a halfpower law. The introduction of this momentum length scale

$$
l_{m}=\frac{M_{o}}{U_{\infty}^{2}}=w R^{2}
$$

in which $M_{o}$ is the initial kinematic momentum flux $U_{\text {jet }}^{2} w, R$ is the velocity ratio

$$
R=\frac{U_{\text {jet }}}{U_{\infty}}
$$

and $w$ is the width of the planar-jet slot in the streamwise direction, enabled them to derive the following expression to model the centerline of the trajectory for the nonbuoyant case:

$$
y=c_{j} R \sqrt{w x}
$$

with $c_{j}$ being a constant of proportionality found to have a typical value of 1.2 in their studies.

Linear jets issuing into quiescent air spread with a half-width of $b_{1 / 2}=0.11 s$, with $s$ being the distance along the centerline of the jet from the exit slot. However, planar jets in a crossflow experience significantly different flow conditions due to the coflowing ambient velocity along the outer edge, the strong curvature along the edge 


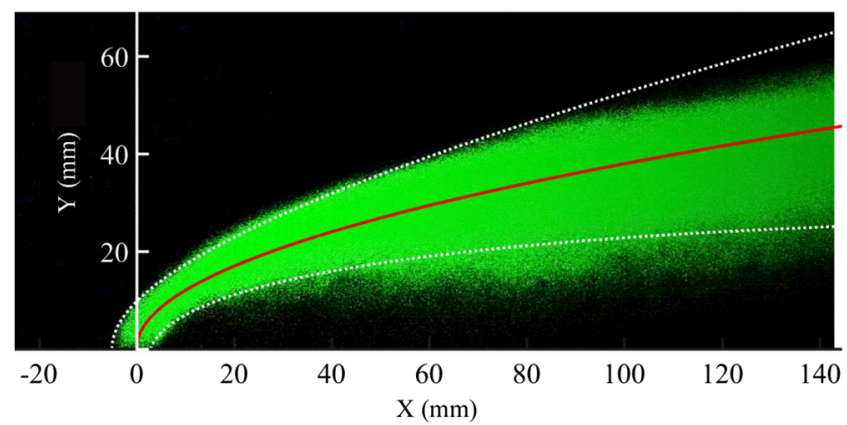

Fig. 1 Flow visualization of a single fluidic spoiler; $U_{\infty}=U_{j}=50 \mathrm{~m} / \mathrm{s}$ $(R=1)$ and $w=100 \mathrm{~mm}$.

trajectories, and the consequence of a recirculation zone that can develop on the leeward side of the jet. These three factors affect the stability and entrainment of the inner and outer SLs, and Ramaprian and Haniu [35] found that the planar jet in a crossflow tended to have a larger spreading rate, which they estimated to be

$$
b_{1 / 2}=c_{b} s
$$

in which $s$ is now the curved trajectory, and the proportionality constant $c_{b}$ was found to have values of between 0.12 and 0.15 in their studies.

Advances in laser-based measurement techniques for fluid dynamics have been made, because the Ramaprian and Haniu [35] work and PIV analysis now allow experimental results to be compared to such models. Figure 1 shows an example of flow visualization from a study by Zhao [36] of a single fluidic spoiler (planar jet in a crossflow) on which the curves of Ramaprian and Haniu [35] are superimposed.

The spreading results in a maximum shielding height and a reattachment point of the half-width trajectory on the leeward side. Oerlemans and de Bruin [30] used Eqs. (4) and (5) to find the point of maximum shielding for a value of $c_{b}=\overline{0} .15$, in which their research investigated how the fluidic spoiler might be used to reduce the aerodynamic noise of landing gear. Their results are improved upon here by minimizing the mathematical error in the derivation of terms. As such, the maximum shielding height is derived to be $y_{\max }$ at a streamwise position $x_{\max }$ downstream of the center of the jet slot. The height of the jet centerline trajectory at this point is $y_{c}$ :

$$
x_{\max }=15.6251 w R^{2} \quad y_{\max }=2.2617 w R^{2} \quad y_{c}=4.7434 w R^{2}
$$

This analysis demonstrates that the maximum shielding height is just under $50 \%$ of the jet centerline height at this point, and occurs at a location downstream of approximately seven times the maximum shielding height. What is important to note here is that the shielding height is proportional to both the slot width and the square of the velocity ratio. In summary, however, despite the improvement described here and through observation of Fig. 1, the authors have found that the Ramaprian and Haniu [35] model matches well the curvature of the jet close to the outlet, but, being a quadratic curve, the recirculation is not well captured further downstream. This is particularly evident in Fig. 1 when comparing the centerline of the real flow to the theoretical curve.

\section{B. Numerical Analysis}

To explore an alternative method to model the trajectory of a planar jet in a crossflow, which might better include the effect of the recirculation zone, a 2-D steady numerical analysis was performed. The numerical domain is shown in Fig. $\underline{2}$ with vertical side boundaries set to be translational periodic. The governing equations were formulated to eliminate all gradients in the spanwise direction by taking only one node in this direction. Using this approach, a 2-D

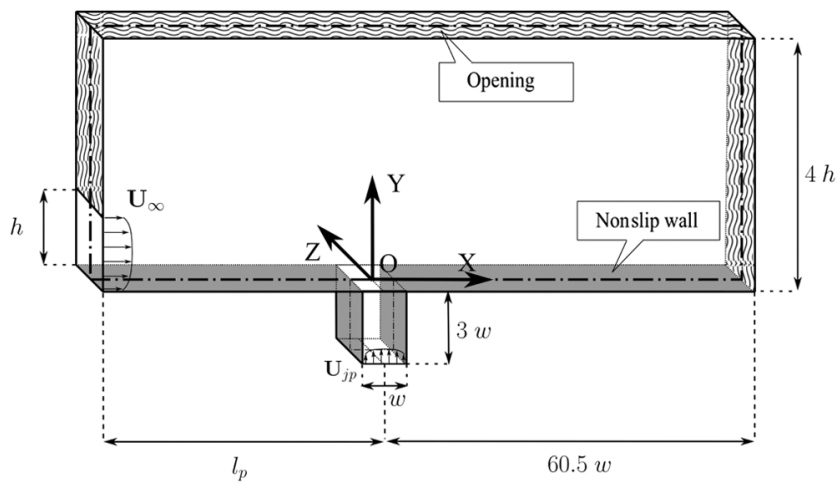

Fig. 2 Numerical domain showing boundary conditions and dimensions. Not to scale.

plane of interest can be examined at a relatively low computational cost, in which this plane in question will be seen to correspond to the PIV laser sheet generated in the experiments. The downstream length of the domain was chosen to be equivalent to 60 times the width of a $10 \mathrm{~mm}$ jet slot, which was found to be sufficient to include downstream effects of the trajectory. The simulation represents a planar jet issuing into a crossflow with the height $h$ of the crossflow significantly greater than the width $w$ of the jet. With only one boundary surface being solid having a no-slip boundary condition, this allows the planar jet to redirect the crossflow over its trajectory. The physics might represent, for example, a fluidic spoiler being discharged from an aircraft fuselage. In these tests, a value of $h=75 \mathrm{~mm}$ was chosen, as this allows the analysis to be compared to experimental results to be found in the literature [37], in which $h$ is the height of an open jet grazing over a surface providing the crossflow. In accordance with similar works, an inlet length for the planar jet of $3 w$ was incorporated to allow important flow features at its exit to be captured. A shear-stress-transport turbulence model was used. The meshed numerical domain has 141,576 grids in total with a refinement of the domain resulting in a greater node density in the region of the slot. These mesh parameters were chosen after a mesh sensitivity analysis was performed and results were found to converge with increasing mesh density.

Figure $\underline{3}$ presents a result for the benchmark case of $U_{\infty}=U_{j}=$ $40 \mathrm{~m} / \mathrm{s}(\bar{R}=1)$ and $w=10 \mathrm{~mm}$. The result shows the characteristics of the performance of the fluidic spoiler. The trajectory of the planar jet curves downstream as the crossflow is redirected over it, resulting in a low-velocity shielded area on its leeward side until the flow reattaches further downstream. The objective of this research was to study the pressure reduction of a downstream cylindrical cavity by designing the planar jet, such that the cavity orifice is shielded by this fluidic spoiler.

To characterize the trajectory of the fluidic spoiler, the streamline passing through the center of the slot was identified. This is plotted in both Figs. $\underline{3}$ and $\underline{4}$. Also to be seen clearly in Fig. $\underline{4}$ is the recirculation

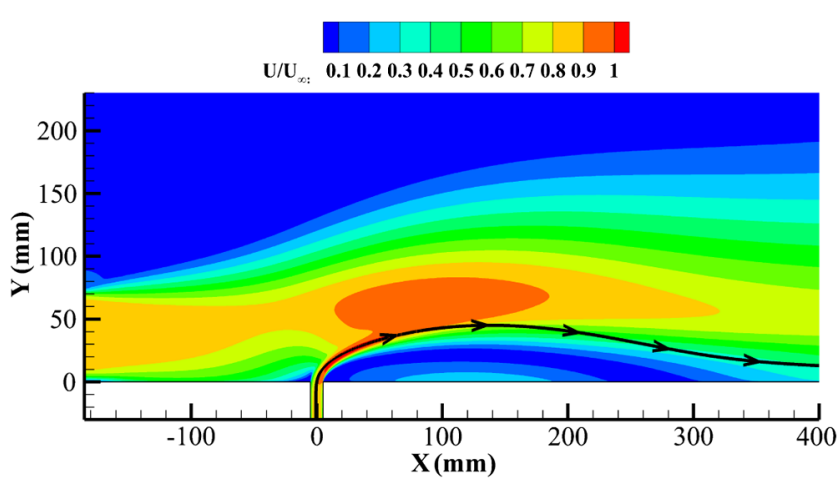

Fig. 3 Numerical normalized velocity result for the benchmark case of $U_{\infty}=U_{j}=40 \mathrm{~m} / \mathrm{s}(R=1)$ and $w=10 \mathrm{~mm}$. 


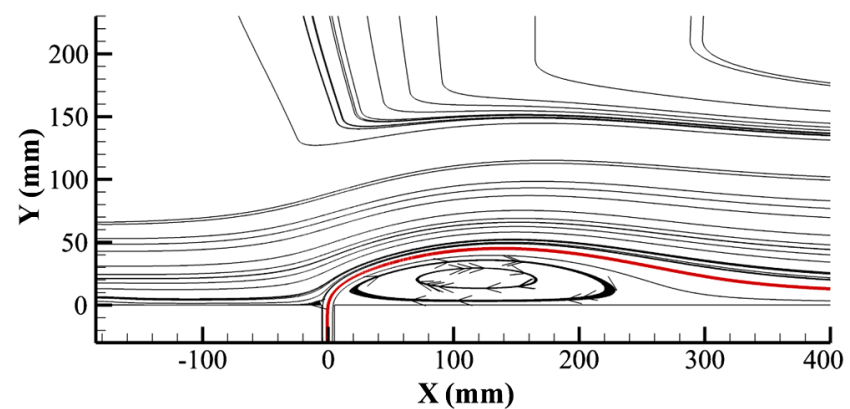

Fig. 4 Streamlines for the benchmark case; the slot center streamline is identified.

zone on the leeward side of the jet. This zone and the centerline, which curves downward toward the surface after reaching a maximum height, are both more accurate representations of the real behavior of the flow. The centerline model in Eq. (4) cannot accurately represent this same behavior. A parametric analysis was performed with the numerical model to determine the dependence of the trajectory on $U_{\infty}, U_{j}, R$, and $w$. Figure 5 shows how the maximum point of the streamline increases with the slot width when the velocities are kept constant, and the height appears to be proportional to approximately $w^{4 / 3}$. The height increase is a result of the increase in momentum of the fluid, and this maximum position occurs successively further downstream with increasing slot width.

Figure 6 shows the response of the planar jet to a change in velocity ratio $R$ for a fixed slot width of $10 \mathrm{~mm}$. This is in effect a study of the increase in jet velocity when all other parameters are kept constant. As to be expected, the maximum height increases with $R$ and is proportional to $R^{2}$.

Figure 7 confirms how the trajectory depends on the velocity ratio $R$, as opposed to the individual magnitudes of either the crossflow or jet velocities.

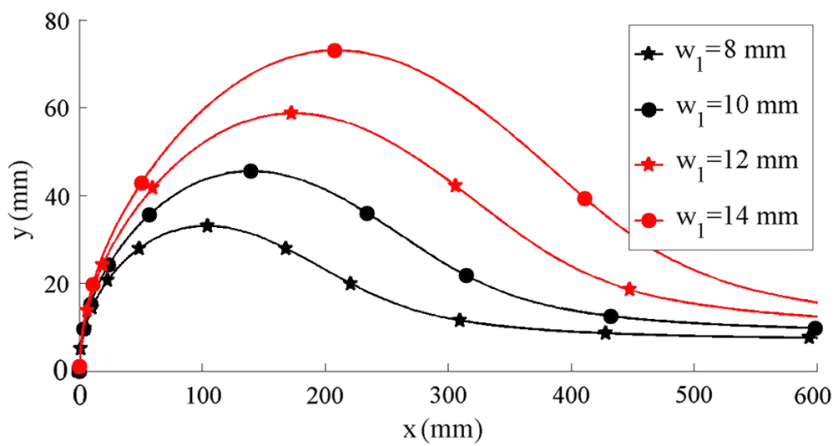

Fig. 5 Numerical analysis; center streamline as a function of slot width $U_{\infty}=U_{j}=40 \mathrm{~m} / \mathrm{s}$.

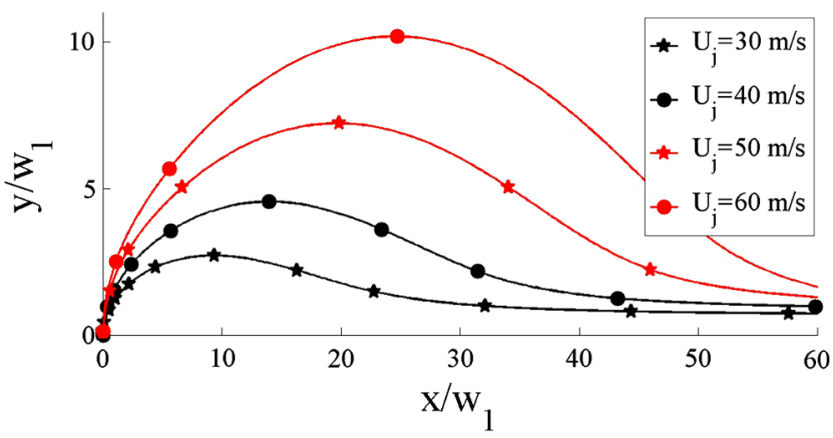

Fig. 6 Numerical analysis; center streamline as a function of $R$. $U_{\infty}=40 \mathrm{~m} / \mathrm{s}, w=10 \mathrm{~mm}$.

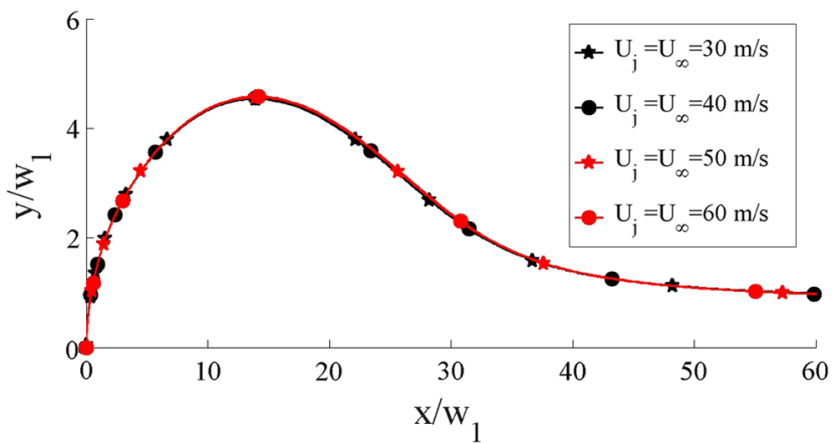

Fig. 7 Numerical analysis; center streamline as a function of $U_{\infty}$ and $U_{j}$ with $R=1 ; w=10 \mathrm{~mm}$.

\section{Experimental Rig Design}

A cavity resonance experiment that incorporates a number of acoustic feedback mechanisms was to be designed and constructed. As the rig was to be built using a relatively low-speed wind tunnel, a preliminary analysis was required to optimally design the cavity given the imposed limitations. In each of the three fluid-resonant oscillations considered in this paper, viz., Helmholtz resonance, longitudinal resonance (plane wave), and azimuthal resonance, the acoustic excitation is assumed to be due to instability in the SL of flow over the cavity opening. The SL excitation frequency can be estimated using the empirical relationship suggested by Rossiter [16]. Given the low Mach number under consideration in the current paper $(M<0.1)$, the upstream-propagating acoustic feedback mechanism usually associated with Rossiter is not expected to occur in the present experiment, because the acoustic frequency corresponding to the cavity opening length would be in the order of $8 \mathrm{kHz}$, far higher than any expected SL oscillation frequency. Rossiter's equation for the SL excitation frequency, however, has been used by many authors to accurately model other feedback mechanisms $[\underline{38}, \underline{39}]$. This equation is given as

$$
S t \equiv \frac{f L}{U} \approx \frac{n-\alpha}{M+(1 / \kappa)}
$$

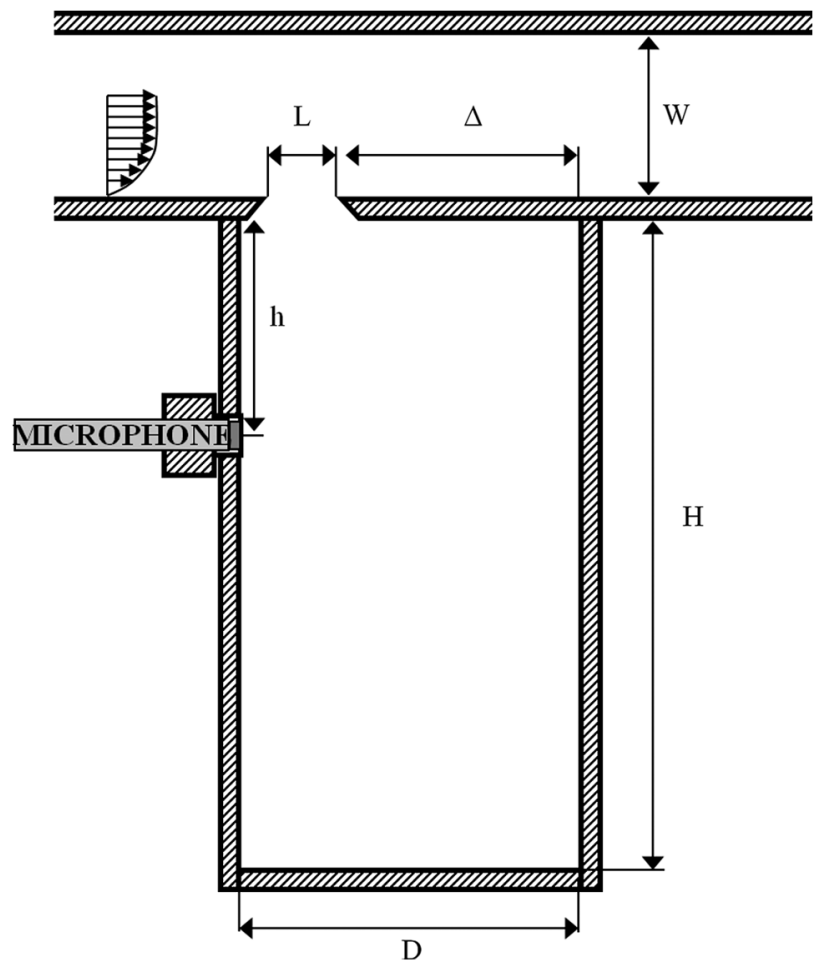

Fig. 8 Schematic of a generic wind-tunnel test section and the cylindrical cavity. 
in which $\alpha$ describes the phase delay; $\kappa$ is the convection velocity of the SL normalized by the freestream velocity; and $n=1,2,3, \ldots$ is the order of the SL mode. For the low subsonic speeds considered here, $\alpha=0$ was found to be appropriate and has been used by other authors $[38,40,41]$, who argued that there is no need to consider a phase delay when the convection speed is much less than the speed of sound. The typically used value of $\kappa=0.5$ is determined from the average of the freestream velocity and the flow in the cavity, and does not include the effects of the boundary layer, which would act to retard the apparent freestream velocity. Over the years, Eq. (7) has been subject to small changes introduced after analytical developments: see, for instance, Heller and Bliss [42] and Howe [43].

As can be seen in Eq. (7), decreasing the characteristic length of the cavity opening, $L$, increases the excitation frequency for a given flow speed. Similarly, from knowledge of duct acoustics and previous work [44], increasing the cavity diameter will result in lower cut-on frequencies for the higher-order modes. Thus, for the low tunnel speeds available, a large diameter and short orifice opening were required to achieve the test objectives allowing excitation of the three different fluid-resonant categories considered.

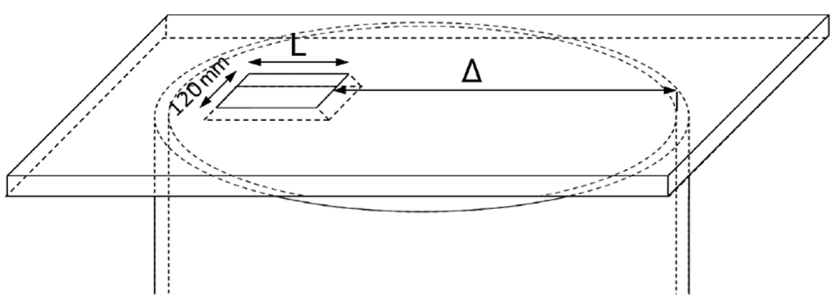

Fig. 9 Schematic of the proposed cavity opening.

\section{A. Numerical Analysis: Wave Expansion Method}

To characterize the acoustic behavior of a proposed experimental rig (cavity and wind-tunnel test section), a numerical simulation was performed. A highly efficient finite difference method originally introduced by Caruthers et al. [45] was used for the analysis. The approach uses wave functions, which are exact solutions of the governing differential equations. The wave expansion method (WEM) in-house code used for this study was developed by Ruiz and Rice [46], and has been examined by Bennett et al. [47] for its applicability in ducts. The schematics of a generic wind tunnel/cavity and opening setup can be seen in Figs. $\underline{8}$ and $\underline{9}$. A number of simulations were performed to finalize the dimensions for the streamwise dimension of the orifice: $L$, the depth/height $H$ of the cavity, and the diameter $D$ of the cylindrical cavity.

A three-dimensional unstructured mesh encompassing the generic wind-tunnel test section and the cavity was generated with commercial software, resulting in approximately 320,000 tetrahedral elements (Fig. 10). In a landing-gear technical report by Bliss and Hayden [48], the cavity-oscillation process for landing-gear wheel bays has been approximated by monopole radiation from the center of the cavity opening. In accordance with this, in this work, a numerical monopole volume source was located at the orifice opening midpoint to simulate an oscillation in the SL. The complex pressure was solved in the domain as a function of source frequency, and the amplitude was plotted on the mesh to give an indication of the pressure field in the cavity/wind-tunnel rig setup. To identify the frequencies at which the resonant modes in the cylinder are at a maximum, the transfer function between the source and the node in the domain, which has the greatest magnitude at any particular frequency, was calculated. The solution for the frequency range of interest is shown in Fig. 11. In this frequency range, 12 different

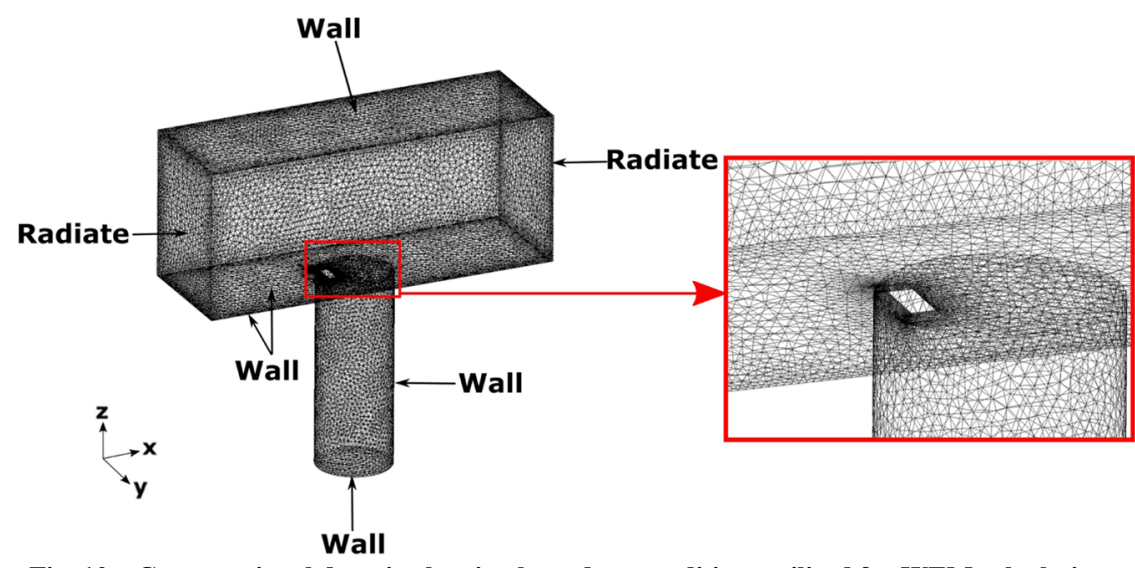

Fig. 10 Computational domain showing boundary conditions utilized for WEM calculations.

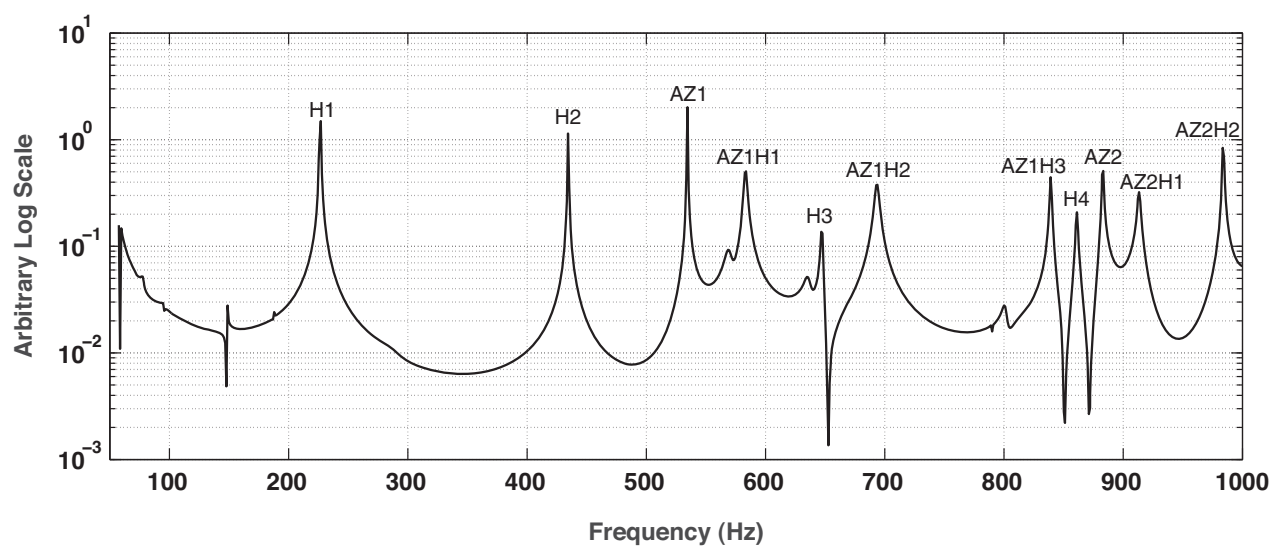

Fig. 11 Excitation frequency of the first 12 acoustic modes (WEM). 


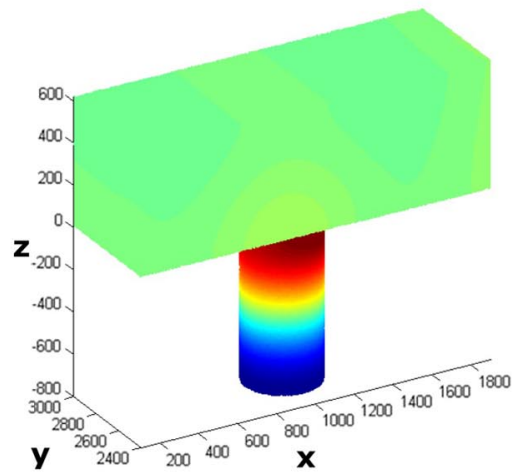

a) $\mathrm{H1}$

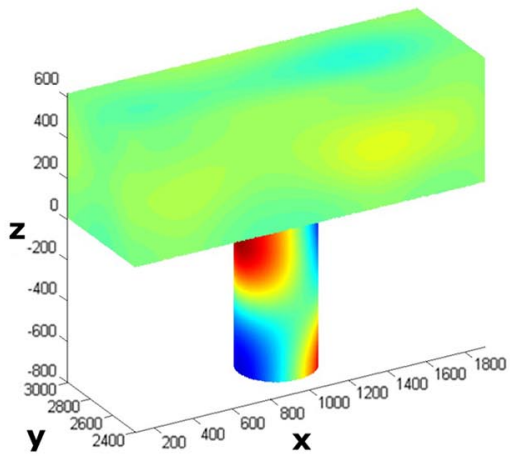

d) AZ1H1

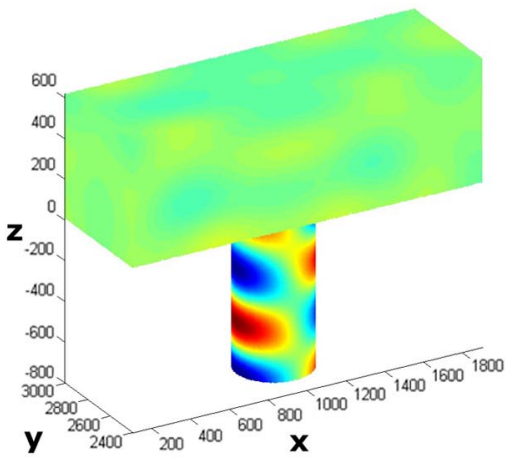

g) $\mathrm{AZ1H3}$

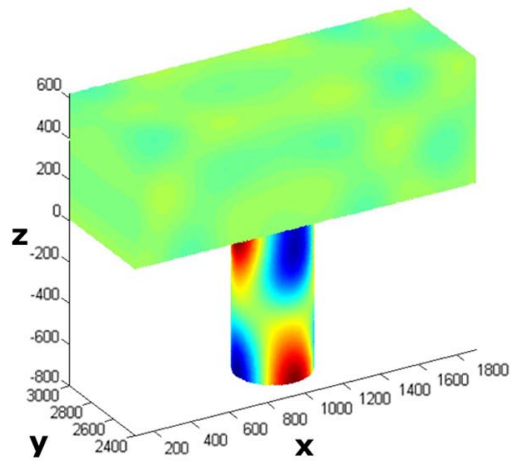

j) AZ2H1

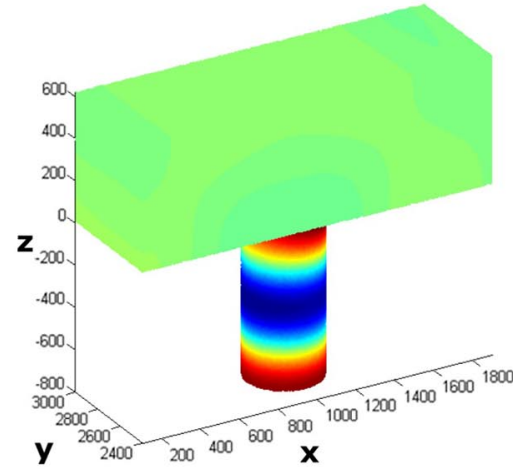

b) $\mathrm{H} 2$

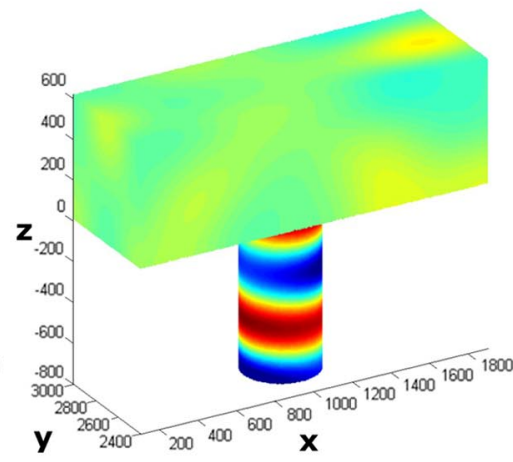

e) $\mathbf{H 3}$

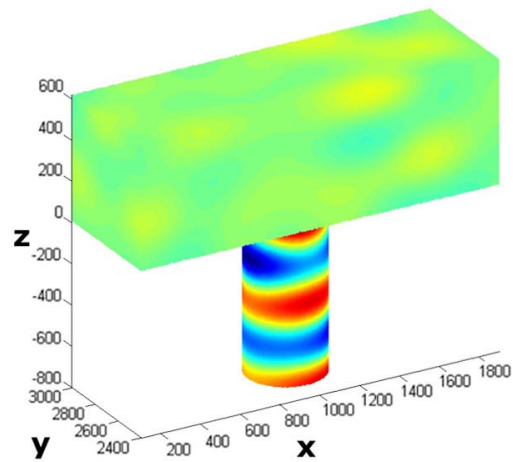

h) $\mathrm{H4}$

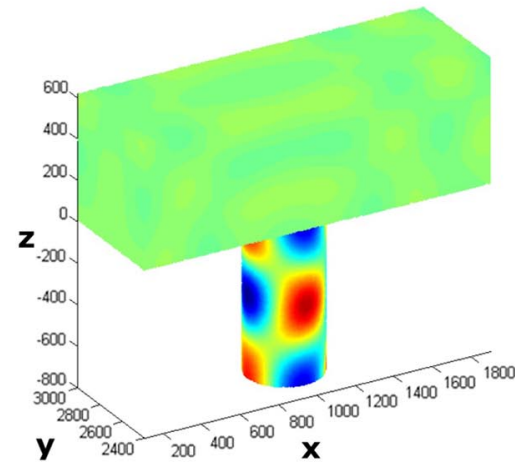

k) $\mathrm{AZ2H} 2$
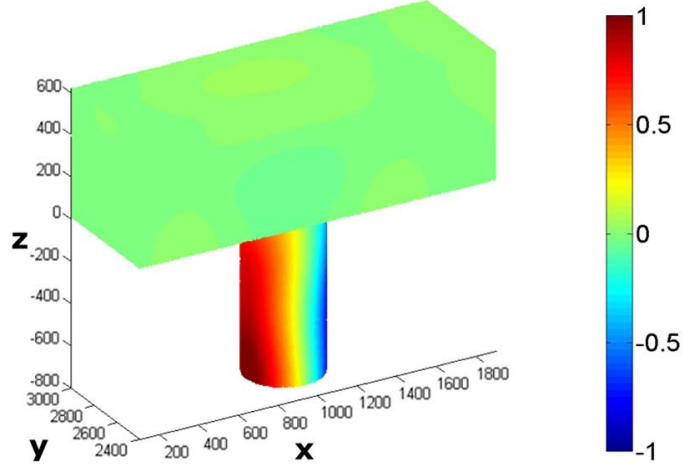

c) AZ1

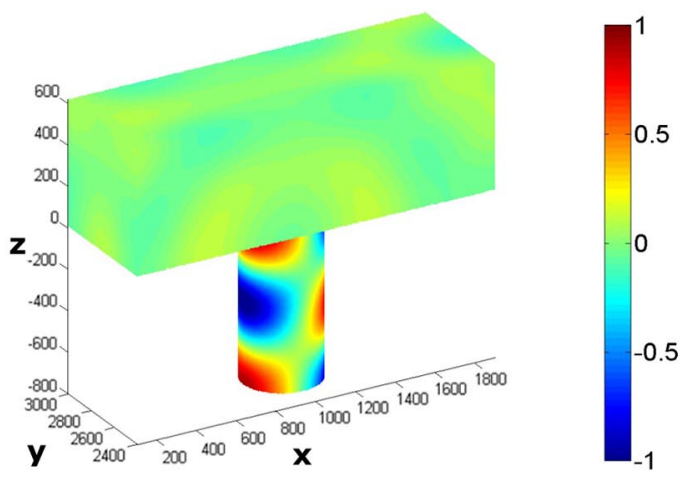

f) $\mathrm{AZ1H} 2$
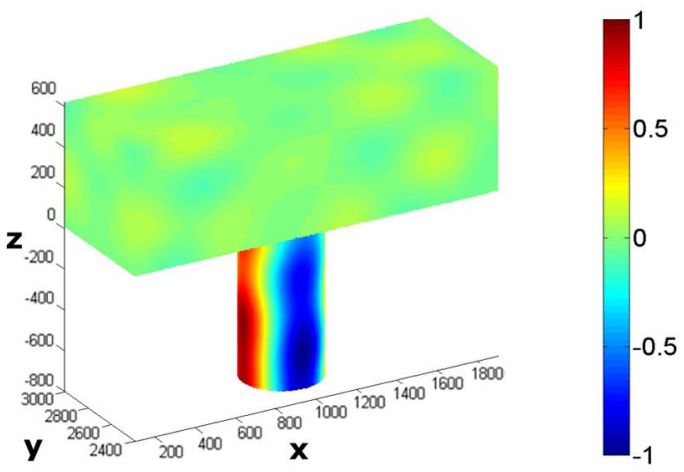

i) $\mathrm{AZ2}$

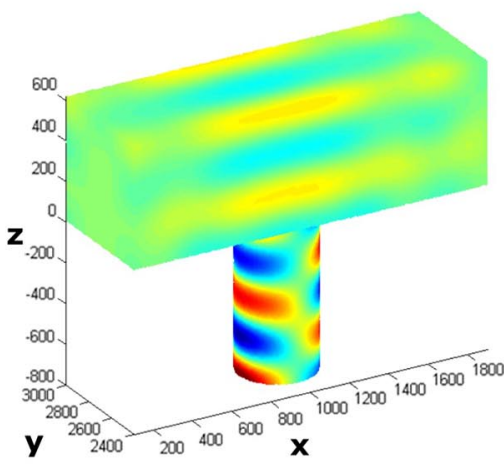

i) $\mathrm{AZ2H3}$

Fig. 12 Numerical analysis. Acoustic mode shapes at the first twelve resonances.

acoustic modes were found, the individual pressure fields of which are shown in Fig. 12.

The frequencies chosen illustrate the first four plane-wave depth/ height or longitudinal modes ( $\mathrm{H} 1, \mathrm{H} 2, \mathrm{H} 3$, and $\mathrm{H} 4)$, which can be approximated as multiples of the half-wavelength corresponding to the internal length of the cavity (cylinder); the first two azimuthal modes, AZ1 and AZ2; and also the first six combination azimuthal- height modes (e.g., AZ1H1), which have effectively received no attention in cavity acoustics. In fact, with respect to aeronautics, typically only the first or second height mode resonance is considered when designing to avoid aeroacoustic cavity noise, such as from burst-disk cavities or vent holes. However, for much larger volumes, such as landing-gear wheel bays or missile bays, higher-order modes have been shown to be excitable within the velocity range of aircraft 

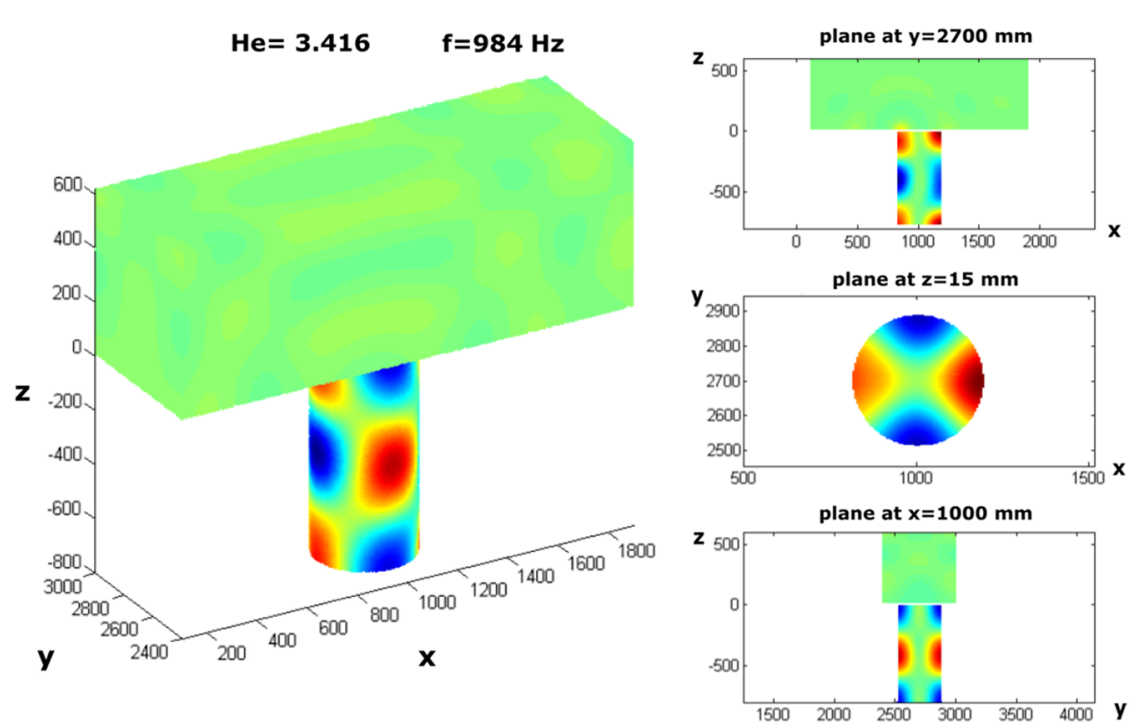

Fig. 13 AZ2H2 acoustic mode showing detailed slice planes through model.

on approach to landing [49-52], and as such, deserve to be examined in the literature.

The common nomenclature in cylindrical duct acoustics is for the subscript $m$ to indicate the azimuthal order and the subscript $n$ to indicate the radial order. Of additional interest in this current study is the measurement of the end reflection from the cavity, which causes the longitudinal standing wave and its superposition on the azimuthal/radial modes, and thus, a third subscript $q$ is used to indicate the depth-mode order. For example, $A_{m, n, q}=A_{0,0,1}$ is the first half-wavelength depth/longitudinal mode, and is also named here as the $\mathrm{H} 1$ mode given that $H$ is the depth of the cavity as per Fig. 8. The first azimuthal mode is termed AZ1 or $A_{m, n, q}=A_{1,0,0}$, and in Fig. 13, the combination mode AZ2H2, $A_{m, n, q}=A_{2,0,2}$, is illustrated in more detail. No radial modes were excited in the range of wind-tunnel speeds tested in the subsequent experimental campaign, and therefore, the results presented here will all be for $n=0$ (i.e., $A_{m, 0, q}$ ). In this work, the frequencies at which the cylindrical duct modes cut on are calculated by

$$
f_{m, n, q}=\frac{\omega}{2 \pi}=\frac{c}{2 \pi} \sqrt{\left(\frac{H e}{R_{c}}\right)^{2}+\left(\frac{q \pi}{H}\right)^{2}}
$$

in which $R_{c}$ is the radius of the cylinder; $q$ is the order of the height mode; and $H e$ or term $k R_{c}=\left(2 \pi f R_{c}\right) / c$ is the dimensionless Helmholtz number, which expresses the cut-on point of each independent higher-order azimuthal/radial mode.

Note that the WEM is poor at predicting the Helmholtz resonance, it being the result of a compressibility effect and is absent from Fig. 11. However, the expected resonance frequency can instead be calculated using Eq. (1) and variations thereof.

Based on the results of the analysis in this section, this generic cavity geometry could allow all three fluid-resonant category configurations to be excited in a cavity of diameter $0.3795 \mathrm{~m}$ by a cavity orifice of $40 \mathrm{~mm}$ in length for the flow range of the wind tunnel available for the tests.

\section{B. Notre Dame Wind-Tunnel Facility and Test Section}

The tests were performed at the Institute for Flow Physics and Control at the University of Notre Dame. A drawdown wind tunnel with an elliptical bell-mouth inlet was used with a $0.6096 \times$ $0.6096 \mathrm{~m}$ test section $\left(2 \mathrm{ft}^{2}\right)$ with a length of $1.83 \mathrm{~m}$. The tunnel inlet consisted of flow management screens and a contraction located upstream of the test section, which result in turbulence levels of the order of $0.5 \%$ or lower. Downstream of the test section, a diffuser decelerates the flow and leads to the primary fan. The floor of the test section is the upper wall of the resonator. The cylinder used was 16 in. schedule 40 PVC, which provides an internal diameter of $0.3795 \mathrm{~m}$ and was sized to have an internal height of $0.8 \mathrm{~m}$. The orifice was machined from $12.7 \mathrm{~mm}(1 / 2 \mathrm{in}$.) aluminum plate, and measured $40 \mathrm{~mm}$ in the streamwise direction and $120 \mathrm{~mm}$ in the spanwise direction, and was machined to have sharp chamfers at $45 \mathrm{deg}$ at the leading and trailing edges; see Fig. 14.

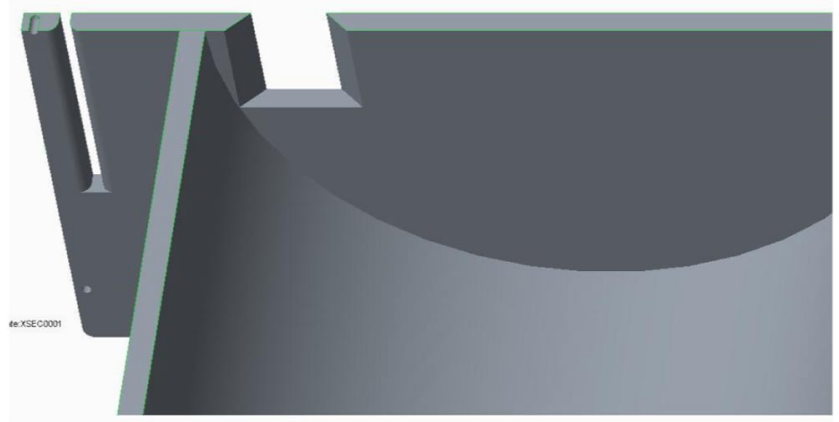

Fig. 14 Solid model of the cavity orifice and slot.

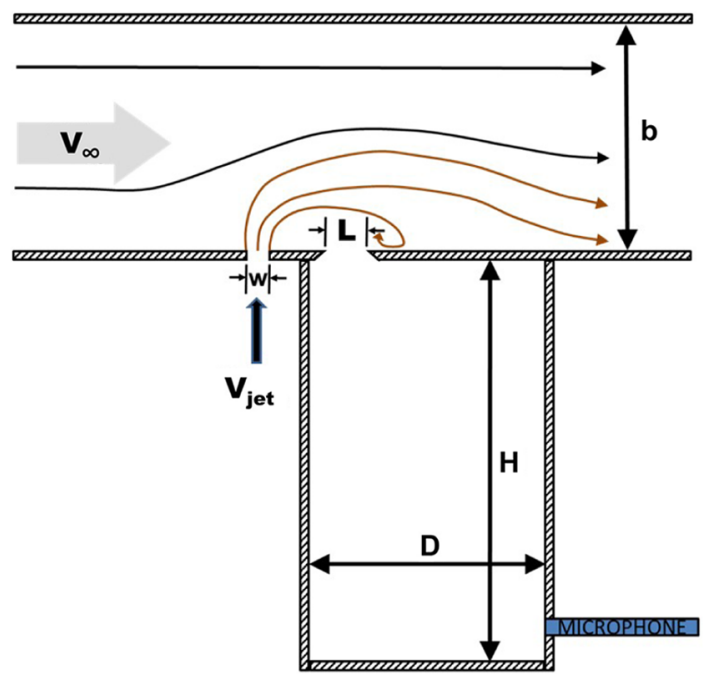

Fig. 15 Schematic of the wind tunnel test section and the cavity. 
The schematics of the test section, which include the slot for the upstream planar jet, are given in Figs. 15 and 16. The planar-jet slot has a 6.35 -mm-wide outlet with a rounded internal geometry to improve the jet development. The spanwise length was $254 \mathrm{~mm}$ initially, but could also be shortened. Based on the WEM analysis, a B\&K microphone was located as per Fig. 15, so that it was not on a nodal line and would, therefore, be sensitive to all acoustic modes in the test frequency range. For the pressure data acquisition, the sampling frequency was $20,000 \mathrm{~Hz}$; the number of samples was $2,097,352$; the Welch method was used to compute the autospectral density functions; the number of samples per fast Fourier transform was 4096; a Hamming window was applied; and a 75\% overlap was used. A pitot-static tube was used to measure the planar-jet exit velocity and to monitor the tunnel speed $U_{\infty}$. A PIV system was installed for flow visualization and will be used for the full velocity analysis in future work.

\section{Response of the Resonator to a Grazing Flow}

\section{A. Baseline Opening}

Without the planar jet turned on and with its slot covered, a velocity sweep of the tunnel in increments of $1 \mathrm{~m} / \mathrm{s}$ was performed by controlling the axial fan motor speed. The results of sound pressure (measured in $\mathrm{dB}$ ) at the microphone location are presented in Fig. 17 superimposed with the theoretical information from Eqs. (1 $)$ and $(\underline{8})$ for the duct resonances, and Eq. (7) for the SL excitation curves. Frequencies are given in nondimensional form (Helmholtz number $=2 \pi R_{c} f / c$ ). As Verdugo et al. [53] stated in their work, and in agreement with other authors [44], the convection velocity constant can be measured or is more often chosen as a best fit to the experimental results. In the literature, a wide range of studies can be found for cylindrical cavities whose aspect ratios and opening characteristics can vary significantly, with the values of $\kappa$ varying in turn. In this work, values of $\alpha=0$ and $\kappa=0.32$ are used, having

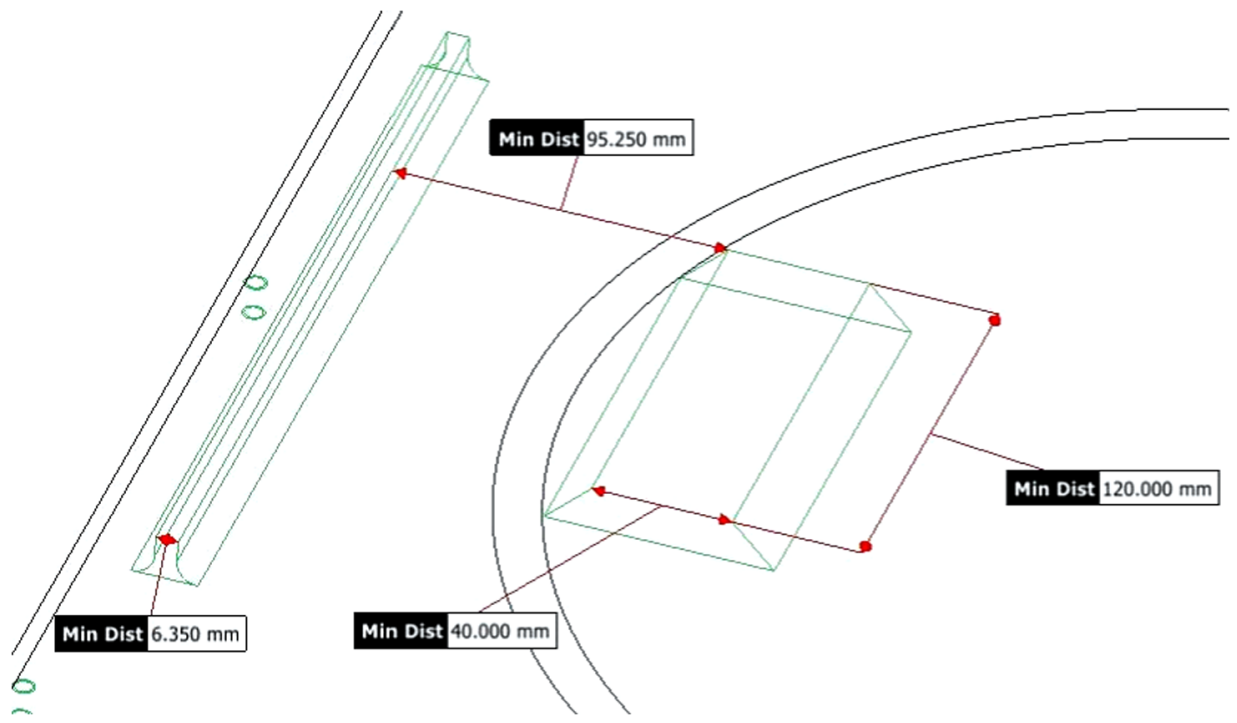

Fig. 16 Geometry of the openings; $\min$ dist $=$ minimum distance.

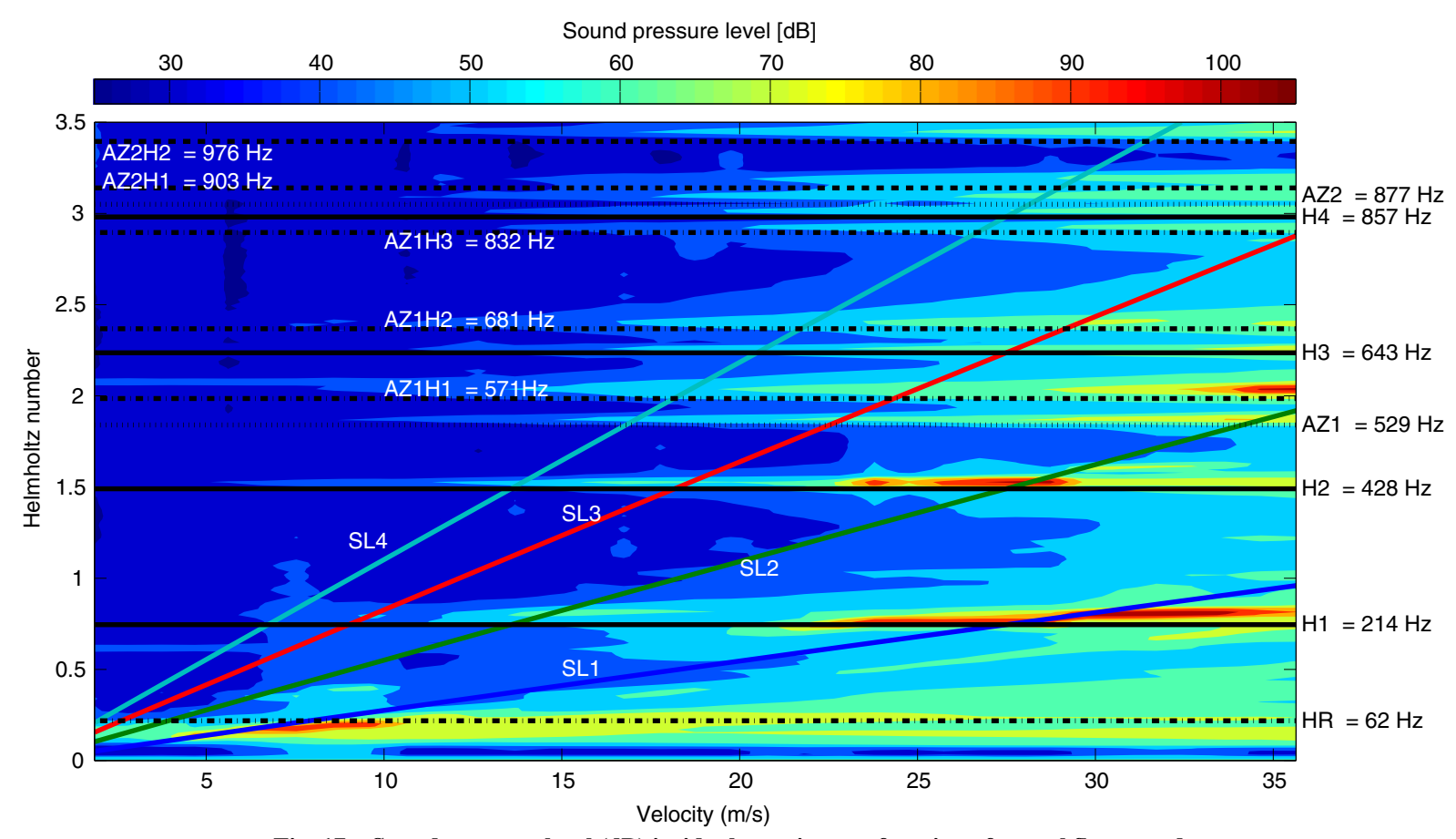

Fig. 17 Sound-pressure level (dB) inside the cavity as a function of tunnel flow speed. 
been found to be a best fit to the measured data. The results show that the SL instabilities are able to excite a number of different modes in the cavity. The first SL mode strongly excites the Helmholtz resonance in the range from 5 to $11 \mathrm{~m} / \mathrm{s}$, and it is seen that the theory of Eq. (1) accurately predicts the resonance frequency with the effective slug length $l_{s}$ set equal to the spanwise dimension $L$. At higher flow speeds, the first SL mode also strongly excites the first plane-wave longitudinal mode $\mathrm{H} 1$. A common characteristic of such resonance phenomena is that "lock on" can occur prior to coincidence and maintain postcoincidence. This is seen in the plot where the resonance occurs for a range of velocities, which is greater than simply the point of intersection of the horizontal and diagonal lines. Verdugo et al. and Stephens et al. discussed this point in depth for the resonance of cylindrical cavities [20,21,54].

In addition to the second SL exciting the second depth mode $\mathrm{H} 2$, of particular interest in this plot is evidence of the excitation of the azimuthal mode AZ1 and of the very high resonance of the combined mode AZ1H1 at a Helmholtz number just above $\mathrm{He}=2$. These modes have seen almost no attention in the literature, but yet can be excited under the correct conditions and, for large-volume landinggear wheel bays, can be excited within the velocity range of a landing aircraft [49]. There is also evidence of the second combination mode AZ1H2 being excited at $H e=2.37$ by both the second and third SL modes. AZ2; H4; and the higher-order combination modes AZ1H3, $\mathrm{AZ2H} 1$, and $\mathrm{AZ2H} 2$ are also all excited, albeit at lower magnitudes, at the higher wind-tunnel velocities.

The frequencies of the numerically determined modes are provided in Table 1 . Small but systematic differences can be noticed when they are compared with the analytical values, which assume a closed-closed cylinder with no orifice. These differences are caused by the distortion of the pressure pattern within the cylinder. The pressure reduces in the test section, which induces a pressure adaptation in proximity to the orifice. The effects of the opening are similar to a reduction of the cavity depth [44] and result in the frequencies of the modes being higher than the theoretical values.

The excitation by the orifice SL of the closed-test-section wind tunnel was also examined. A numerical analysis using the WEM code verified that, as expected, wind-tunnel duct modes could be excited over a wide range of frequencies. However, none of these were measurable at any significant amplitude by the microphone flush-mounted with the inside surface of the cylinder. In the study by Bennett et al. [44], a number of different orifice size aspect ratios and locations, relative to the central axis of the cylinder, were studied, and the acoustic pressure was measured at a number of different microphone locations. In that work, it was found that, in some instances, a half-wavelength mode resonated between the end of the cylinder and the inside surface of the opposite wall of the wind tunnel. No such excitation is measured in this current work.

\section{B. Effect of Fluidic Spoiler}

One of the objectives of this research was to examine how a planar jet might be used to attenuate the cavity resonances caused by SL excitation. Figure 18 shows a flow visualization of the planar jet at a jet velocity of $\overline{U_{j}}=10 \mathrm{~m} / \mathrm{s}$ in a crossflow of $U_{\infty}=8 \mathrm{~m} / \mathrm{s}$ $(R=1.25)$. The jet flow was seeded, and the laser sheet was parallel to the flow at the midpoint of the span/orifice/slot. Figure 18 shows a single still shot from the PIV. To compare to the theoretical and steady computational fluid dynamics (CFD) analysis of Secs. II.A and II.B,

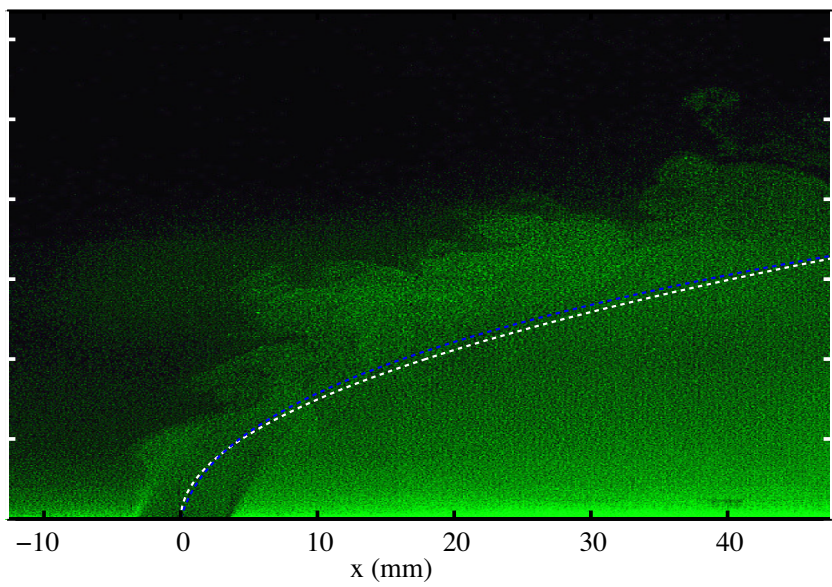

Fig. 18 A Single still from the PIV showing the planar jet.

the theoretical curve of Eq. (4) was superimposed onto the image. Values of $c_{j}=1.0$ and $c_{b}=0.2$ were chosen, which are quite different from those of Ramaprian and Haniu [35], which could be related to the fact that this study is in air. Further PIV work is required to study further downstream of the slot, to study the recirculation zone, and to see if the jet reattaches to the wind-tunnel floor.

Figure 19 presents again the contour plot for the tunnel velocity sweep, but on this occasion, the planar jet is active with an exit velocity of approximately $11 \mathrm{~m} / \mathrm{s}$. The velocity ratio $R$ in this plot ranges from approximately 11 down to 0.3 . The results are very positive. Very significant resonance amplitude reductions are measured. Interestingly, the Helmholtz resonance, while decreasing in amplitude, has also dropped in frequency and is excited for a much larger velocity range than with no fluidic spoiler. The drop in frequency could be due to the fact that the effective slug length $l_{s}$ can no longer be approximated to be equal to the streamwise orifice length $L$. In addition, the unsteadiness of the jet may now be providing broadband excitation of the cavity, resulting in an excitation over a greater velocity range.

To examine the response in more detail, single spectra at specific tunnel speeds where high resonance amplitudes occur are examined with and without the effect of the fluidic spoiler (Fig. 20). Noise reductions of up to $33 \mathrm{~dB}$ can be identified as a result of the passive flow control. Interestingly, at $U_{\infty}=8 \mathrm{~m} / \mathrm{s}$, while a pressure reduction of $15 \mathrm{~dB}$ is achieved at the peak Helmholtz resonance, the pressure response increases for the rest of the frequency range. This only seems to occur for this tunnel speed. It appears as if the fluidic spoiler excites the cavity with low-level broadband energy, which excites the full frequency range, but which is then dominated by the higher-amplitude tunnel flow and recirculating fluidic-spoiler energy at higher tunnel speeds. Also worth noting is that, at $36 \mathrm{~m} / \mathrm{s}$, the mode that resonates the greatest is the AZ1H1 mode, which is a mode rarely examined in cavities. The fluidic spoiler successfully reduces this pressure by $23 \mathrm{~dB}$.

The same analysis was repeated, but at a higher fluidic-spoiler velocity $=16 \mathrm{~m} / \mathrm{s}(16 \geq R \geq 0.44)$. Figure 21 presents the acoustic pressure response as a function of the tunnel velocity. In Fig. 22, the pressure response at the same four specific tunnel velocities is

Table 1 Frequencies $(\mathrm{Hz})$ of the acoustic modes of a cylindrical cavity $(D=0.3795 \mathrm{~m}$ and $H=0.8 \mathrm{~m})$ at $22^{\circ} \mathrm{C}$

\begin{tabular}{lccccccccccc}
\hline \hline & \multicolumn{10}{c}{ Modes } \\
\cline { 2 - 12 } & $\mathrm{H} 1$ & $\mathrm{H} 2$ & $\mathrm{AZ1}$ & AZ1H1 & H3 & AZ1H2 & AZ1H3 & H4 & AZ2 & AZ2H1 & AZ2H2 \\
\hline$(m, n, q)^{\mathrm{a}}$ & $(0,0,1)$ & $(0,0,2)$ & $(1,0,0)$ & $(1,0,1)$ & $(0,0,3)$ & $(1,0,2)$ & $(1,0,3)$ & $(0,0,4)$ & $(2,0,0)$ & $(2,0,1)$ & $(2,0,2)$ \\
Helmholtz number & 0.75 & 1.5 & 1.84 & 1.99 & 2.24 & 2.37 & 2.9 & 2.98 & 3.05 & 3.14 & 3.4 \\
Analytical & 214 & 429 & 529 & 571 & 643 & 681 & 833 & 858 & 877 & 903 & 977 \\
WEM & 226 & 434 & 535 & 583 & 647 & 693 & 839 & 861 & 881 & 914 & 984 \\
\hline \hline
\end{tabular}

${ }^{a}$ Mode order: azimuthal, radial, and longitudinal. 


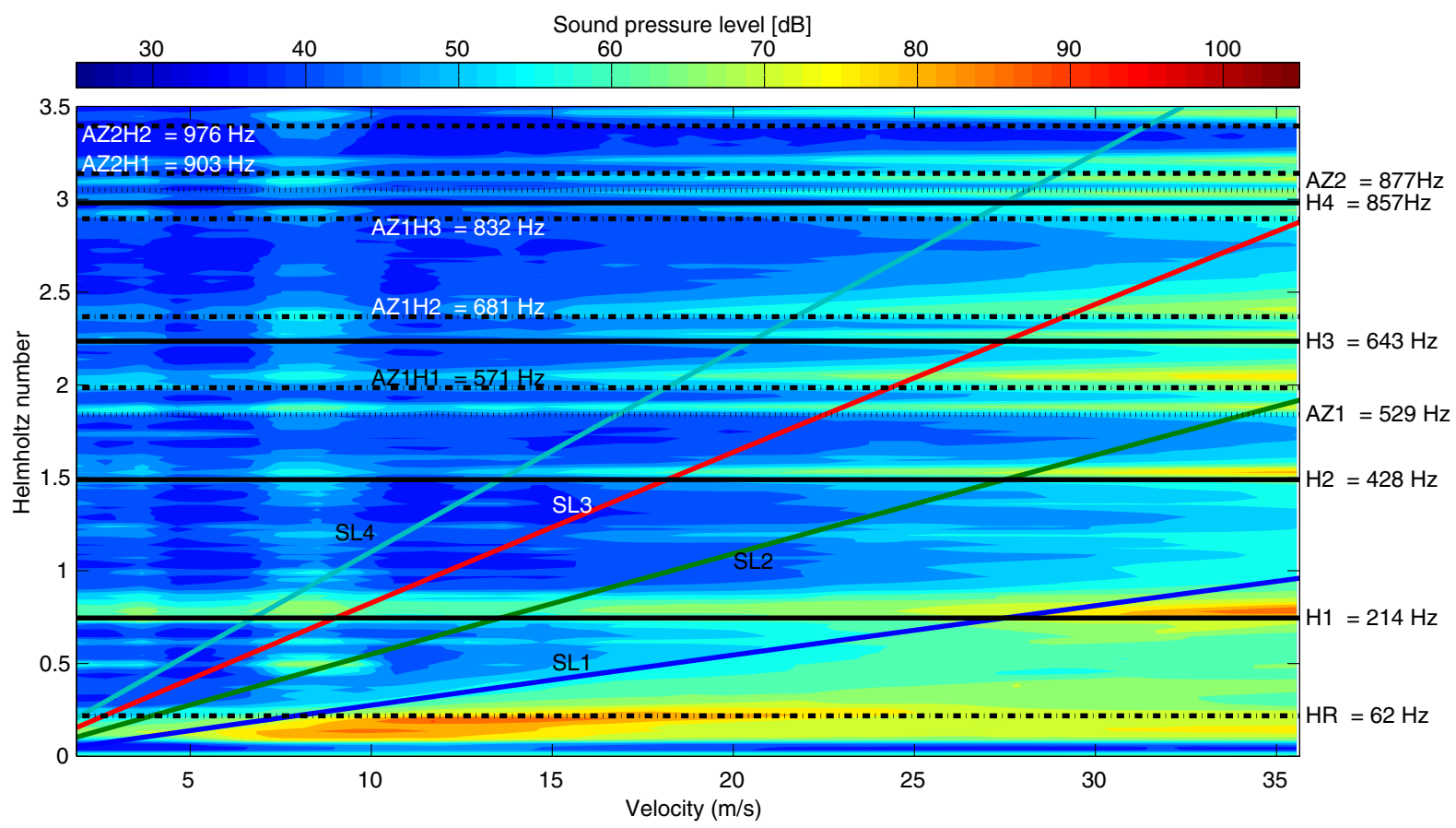

Fig. 19 Sound pressure level (dB) vs tunnel flow-speed. Fluidic spoiler at 11 m/s.

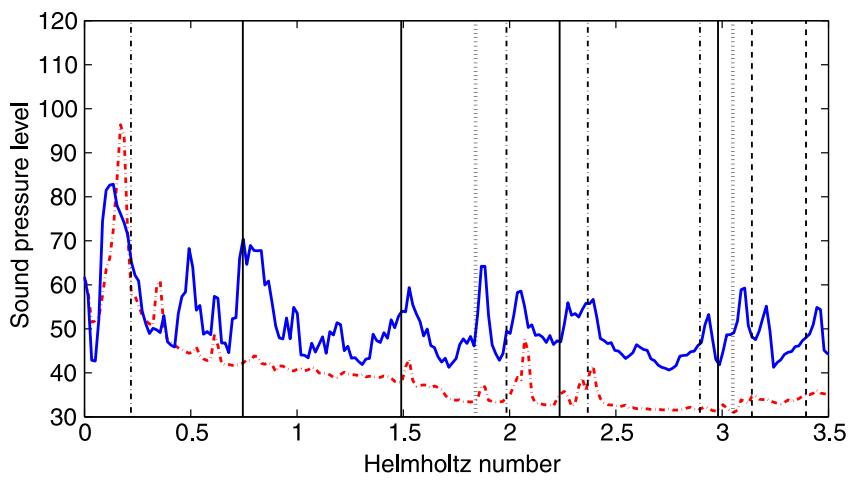

a) $U_{\infty}=8 \mathrm{~m} / \mathrm{s}, \mathrm{R}=0$ (dashed red), $\mathrm{R}=1.38$ (solid blue)

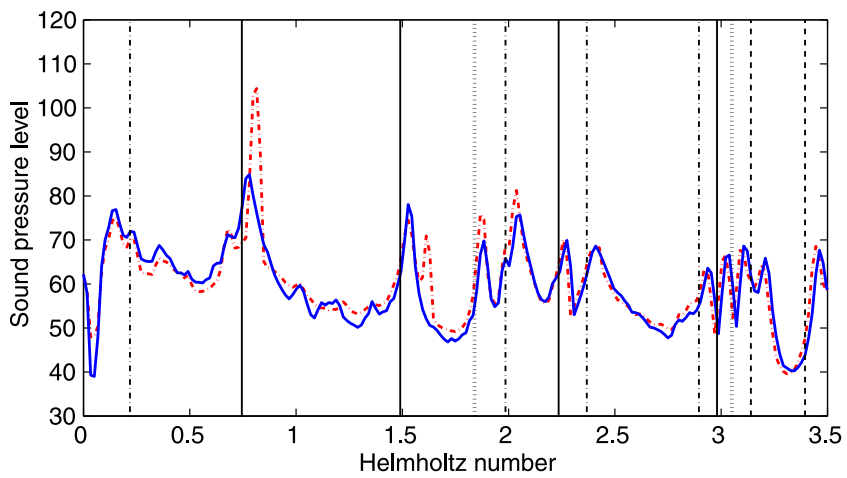

c) $U_{\infty}=33 \mathrm{~m} / \mathrm{s}, \mathrm{R}=\mathbf{0}$ (dashed red), $\mathrm{R}=\mathbf{0 . 3 3}$ (solid blue)

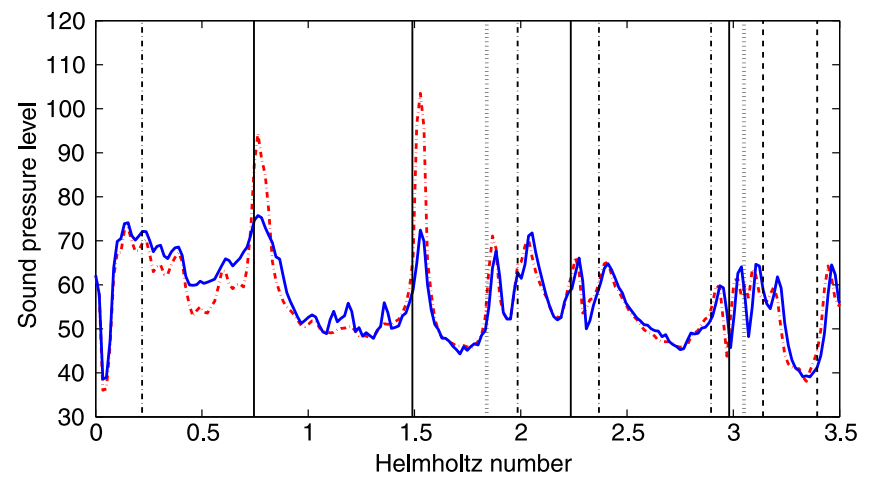

b) $U_{\infty}=29 \mathrm{~m} / \mathrm{s}, \mathrm{R}=0$ (dashedred), $\mathrm{R}=\mathbf{0 . 3 8}$ (solid blue)

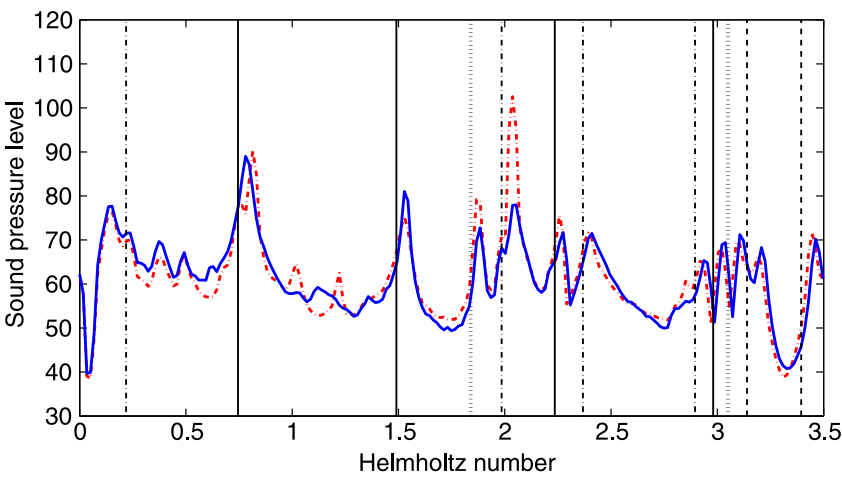

d) $U_{\infty}=36 \mathrm{~m} / \mathrm{s}, \mathrm{R}=0$ (dashed red), $\mathrm{R}=\mathbf{0 . 3 1}$ (solid blue)

Fig. 20 SPL (dB) at specific wind-tunnel velocities. Fluidic spoiler activated at 11 m/s.

presented comparing the results for the two fluidic-spoiler velocities. Surprisingly, the results are effectively identical to each other, and suggest that a minimum threshold velocity is sufficient to suppress the cavity resonance with no further benefit resulting from increased fluidic-spoiler velocities. This is significant, as it suggests that very little additional energy is required to prevent the vortex shedding and to disrupt the SL. A parallel to Mendoza and Ahuja's [55] work, which examined the thickening of the upstream boundary layer, needs to be further investigated.

Figure 23 provides a delta between the baseline case and the $11 \mathrm{~m} / \mathrm{s}$ fluidic-spoiler condition. A positive value (blue color) indicates a noise reduction, whereas a negative value, or red color, indicates a noise increase due to the deployment of the fluidic spoiler. Through a comparison with Fig. 17 , it can be seen that all the main 


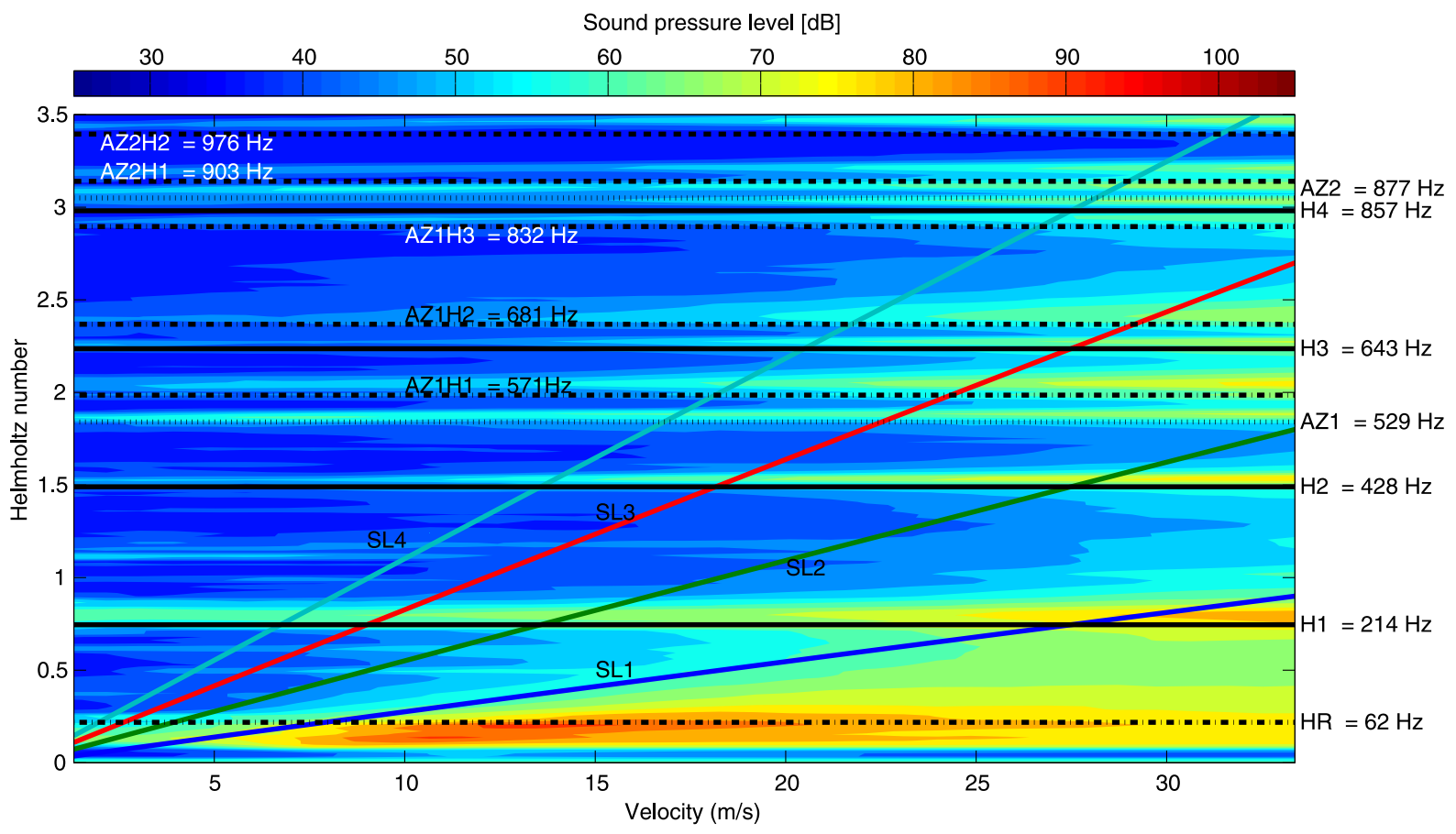

Fig. 21 Sound-pressure level (dB) vs tunnel flow-speed. Fluidic spoiler at 16 m/s.

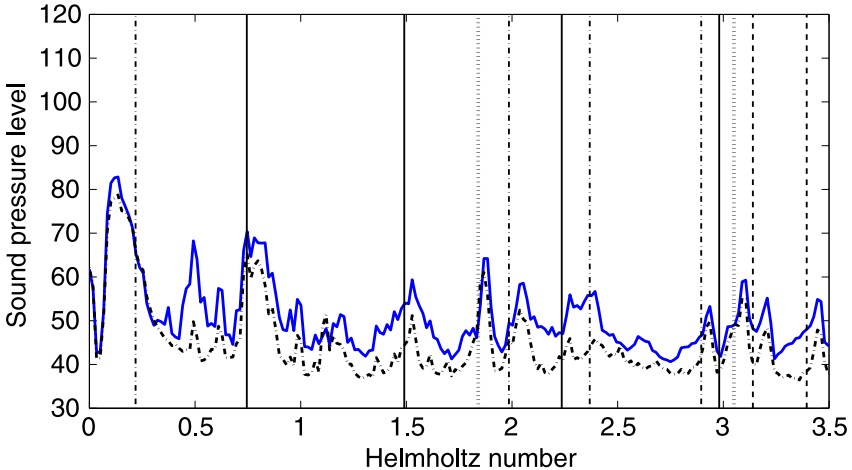

a) $U_{\infty}=8 \mathrm{~m} / \mathrm{s}, \mathrm{R}=2$ (dashed red), $\mathrm{R}=1.38$ (solid blue)

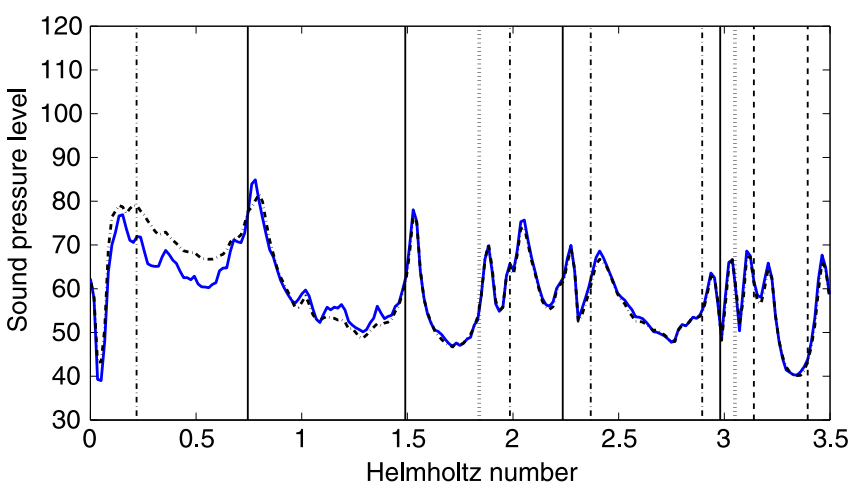

c) $\mathrm{U}_{\infty}=33 \mathrm{~m} / \mathrm{s}, \mathrm{R}=\mathbf{0 . 4 8}$ (dashed black), $\mathrm{R}=0.33$ (solid blue)

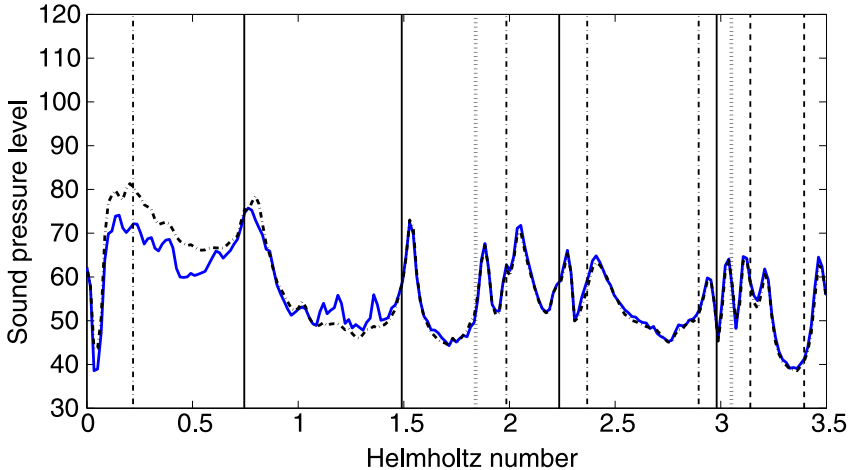

b) $\mathrm{U}_{\infty}=29 \mathrm{~m} / \mathrm{s}, \mathrm{R}=\mathbf{0 . 5 5}$ (dashed black), $\mathrm{R}=\mathbf{0 . 3 8}$ (solid blue)

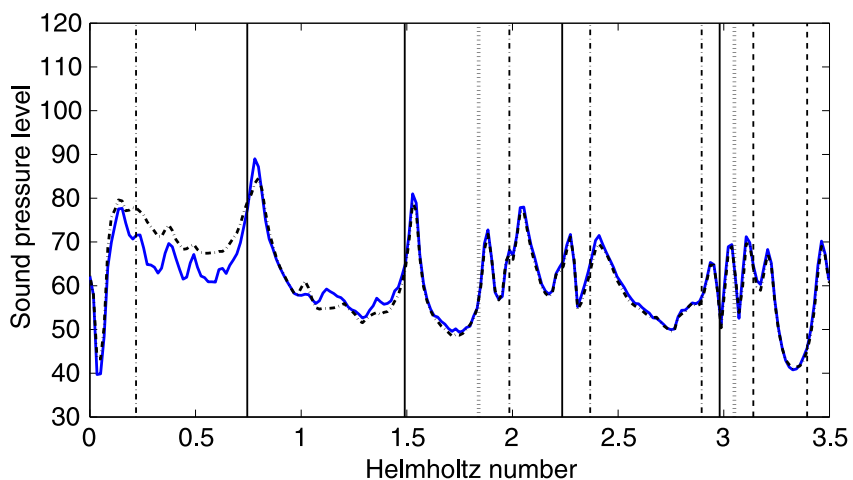

d) $U_{\infty}=36 \mathrm{~m} / \mathrm{s}, \mathrm{R}=\mathbf{0 . 4 4}$ (dashed black), $\mathrm{R}=\mathbf{0 . 3 1}$ (solid blue)

Fig. 22 SPL (dB) at specific wind-tunnel velocities. Fluidic spoiler at $11 \mathrm{~m} / \mathrm{s}$ vs $16 \mathrm{~m} / \mathrm{s}$.

resonance peaks in the cylinder are attenuated, albeit at the cost of some noise increase at low velocities. Interestingly, it can be seen that the majority of the larger noise increases correspond to a velocity ratio $R$ of greater than 1 .

With regard to exactly how much energy, or power, would be required to implement the solution, an analysis could be conducted using the models provided by Zhao et al. [56]. Their work investigated the potential use of a fluidic spoiler as a low-noise technology for landing gear, which could have significant benefits over traditional perforated fairings or meshes. The work examined air-curtain trajectory models and the subsequent generation of aeroacoustic self-noise and slot noise due to the implementation of the fluidic spoiler. A second upstream fluidic spoiler [57] is implemented to successfully reduce the spoiler noise, and an 


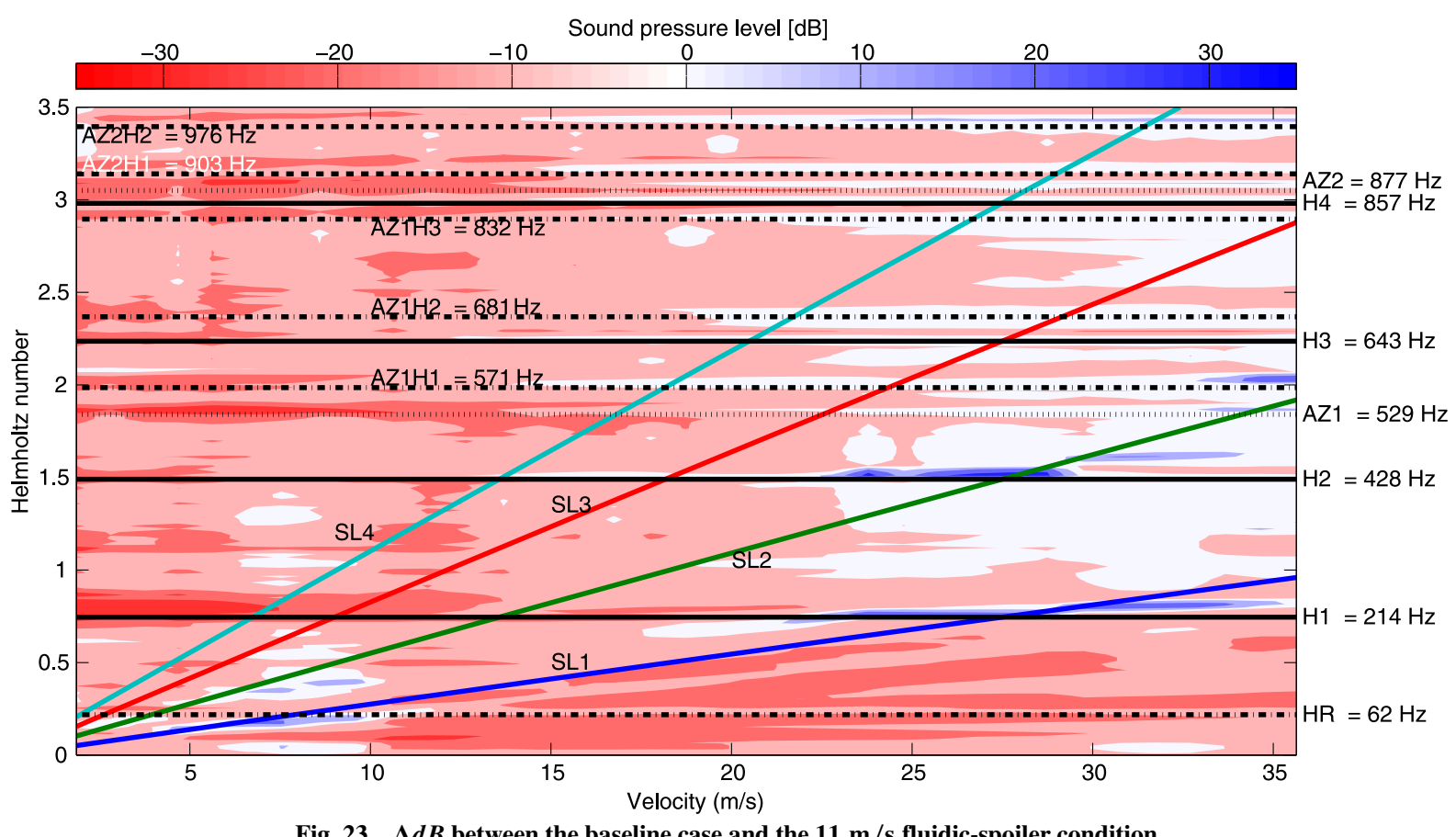

Fig. $23 \Delta d B$ between the baseline case and the $11 \mathrm{~m} / \mathrm{s}$ fluidic-spoiler condition.

estimation of the mass flow required for the shielding of a typical nose landing gear is provided. With regard to the suppression of cavity noise using a fluidic spoiler, it can be stated with reasonable certainty that spoiler noise itself would be of lower amplitude given that the shielding height would be so much less compared to the landing gear. The work by Raman and Raghu [6] can also be referred to for a rough indication of how much energy would be required to modify the SL. In their work, using miniature fluidic devices located on the cavity floor, oscillatory mass injection rates of the order of $0.12 \%$ of the main flow were required to suppress SL-cavity interaction tones. Their innovative work employs a bistable Coanda effect device to spread the flow in the spanwise direction. The current fluidic-spoiler proposal, however, would be expected to require less relative mass flow, as, unlike the aforementioned work, which requires sufficient mass flow to reach the SL from the cavity floor, the fluidic-spoiler jet is issued upstream of the cavity leading edge in the same plane as the SL.

Drag resulting from the implementation of the fluidic spoiler is also to be considered. As the freestream flow in this work is essentially incompressible, future work might use the PIV measurements to quantify the increase in drag from the baseline using a control-volume-type approach. However, this has not been performed to date. Given that the crossflow angle of the fluidic spoiler is, in this case, perpendicular to the main flow, a correction in aircraft trajectory might also be required (depending on amplitudes), which would have knock-on effects with regard to drag or lift. The angle of issue of the fluidic-spoiler jet could be altered to be more acute, but then greater flow rates would be required to obtain the same shielding height. However, for cavity noise suppression, this may again be less of a problem compared to landing-gear noise attenuation given the low shielding height required. The reader is referred to the work of Oerlemans and de Bruin [30] for their investigations on the effect of angle on a single air-curtain trajectory. For practical purposes, quite often an increase in drag can be advantageous, especially for landing aircraft, especially if the airflow used is siphoned off from the engine ducts. Also, a drag increase may be considered to be a small price to pay for the reduction of high-amplitude pressure levels in weapon bays, or the destabilizing recirculation of flow in large cavities or wheel bays. The remaining important point to be made is that the fluidic spoiler can be deployed as needed, unlike fixed spoilers or perforated fairings, and thus, the drag can be eliminated when necessary.

\section{Conclusions}

This paper examined experimentally the use of a fluidic spoiler to suppress the acoustic resonance within a partially closed cylindrical cavity subject to a grazing flow. The work presents a novel method of passive flow control, which consists of an upstream fluidic spoiler, which might also be called an air curtain or simply an upstream planar jet in crossflow. The fluidic spoiler is used to redirect the upstream flow so that the cavity orifice is shielded. This prevents what was the grazing flow from detaching from the upstream leading edge of the cavity, and thus, vortex shedding is suppressed.

A 2-D semi-empirical model from the 1980s is compared to a parametric analysis using computational fluid dynamics (CFD) for a range of velocity ratios. While the model captures well the curvature of the trajectory of the planar jet close to the exit slot, the CFD is shown to be more versatile and captures the full flowfield, including the reattachment of the flow downstream.

A second numerical analysis was performed using a WEM to determine the acoustic behavior of the cavity (in the absence of flow). The analysis allowed the optimum dimensions of the cavity and orifice to be determined for the flow range of the available wind tunnel. An experimental rig was subsequently designed and built. The objective was to have a large volume cavity with a relatively small orifice. This allowed both Helmholtz resonance as well as a variety of standing wave duct modes to be studied. In addition, the cavity, with a diameter/depth ratio of 0.47 , was designed as a cylindrical cavity so that azimuthal and radial modes could be examined. These are modes that are rarely examined in the literature for cavity resonance. Rossiter-type interaction tone frequencies, which are dependent on velocity, were not examined here due to the low Mach numbers available in the closed-section wind tunnel. A microphone flush-mounted with the inside surface of the cavity wall allowed the pressure to be measured at a specific location.

The results from the numerical part of the work allowed a planar-jet slot to be sized and located upstream of the cavity orifice, and PIV allowed for a comparison with the numerical model and verified that the orifice could be shielded by the fluidic spoiler at different velocity ratios.

The initial baseline tests, without the effect of the fluidic spoiler, resulted in large-amplitude resonances in the cavity at frequencies corresponding to the Helmholtz resonance, as well as standing wave resonance along the axis of the cylinder, azimuthally, as well as combinations of the latter two. Excitations of these modes are caused 
by SL oscillations, and result in resonance only in specific tunnel velocity ranges.

Once the fluidic spoiler was deployed, very significant reductions in pressure within the cavity were recorded. Two fluidic-spoiler velocities of 11 and $16 \mathrm{~m} / \mathrm{s}$ were assessed corresponding to a velocity ratio range of approximately $16 \geq R \geq 0.3$ for the windtunnel range tested: approximately $1-36 \mathrm{~m} / \mathrm{s}$. All resonance types were attenuated by as much as $36 \mathrm{~dB}$ in some cases. Little extra benefit seemed to result from the higher-velocity fluidic spoiler, and it seems that very little flow is required to suppress the resonance. This low-energy requirement, as well as the fact that this passive flow control can be easily turned on or off, and therefore, employed as required, makes it an appealing alternative to other such technologies.

\section{Acknowledgments}

The research leading to these results has received funding from The Fulbright Commission in Ireland and CRH plc.

\section{References}

[1] Langtry, R. B., and Spalart, P. R., "DES Investigation of Devices for Reducing Landing-Gear Cavity Noise," 46th AIAA Aerospace Sciences Meeting and Exhibit, AIAA Paper 2008-13, Jan. 2008. doi:10.2514/6.2008-13

[2] Balasubramanian, G., Crouse, B., and Freed, D., "Numerical Simulation of Leakage Effects on Sunroof Buffeting of an Idealized Generic Vehicle," 15th AIAA/CEAS Aeroacoustics Conference, AIAA Paper 2009-3348, May 2009. doi: $10.2514 / 6.2009-3348$

[3] Nakiboglu, G., Belfroid, S. P. C., Tonon, D., Willems, J. F. J., and Hirschberg, A., "A Parametric Study on the Whistling of Multiple Side Branch System as a Model for Corrugated Pipes," Proceedings of the ASME 2009 Pressure Vessels and Piping Division Conference, ASME Paper PVP2009-77754, July 2009, pp. 389-398. doi:10.1115/PVP2009-77754

[4] Aly, K., and Ziada, S., "Azimuthal Behavior of Self-Excited Diametral Modes of Internal Cavities," Proceedings of the ASME 2009 Pressure Vessels and Piping Division Conference (PVP2009-77071), ASME Paper PVP2009-77071, 2009, pp. 139-146. doi:10.1115/PVP2009-77071

[5] Zhuang, N., Alvi, F. S., Alkislar, M. B., and Shih, C., "Supersonic Cavity Flows and Their Control," AIAA Journal, Vol. 44, No. 9, 2006 , pp. 2118-2128. doi: $10.2514 / 1.14879$

[6] Raman, G., and Raghu, S., "Cavity Resonance Suppression Using Miniature Fluidic Oscillators," AIAA Journal, Vol. 42, No. 12, 2004, pp. 2608-2612. doi: $10.2514 / 1.521$

[7] Arunajatesan, S., Kannepalli, C., Sinha, N., Sheehan, M., Alvi, F., Shumway, G., and Ukeiley, L., "Suppression of Cavity Loads Using Leading-Edge Blowing," AIAA Journal, Vol. 47, No. 5, 2009, pp. 1132-1144. doi: $10.2514 / 1.38211$

[8] Yugulis, K., Hansford, S., Gregory, J., and Samimy, M., "Control of High Subsonic Cavity Flow Using Plasma Actuators," AIAA Journal, Vol. 52, No. 7, 2014, pp. 1542-1554. doi:10.2514/1.J052668

[9] de Jong, A., and Bijl, H., "Corner-Type Plasma Actuators for Cavity Flow-Induced Noise Control," AIAA Journal, Vol. 52, No. 1, 2014, pp. 33-42. doi:10.2514/1.J051815

[10] Ukeiley, L. S., Ponton, M. K., Seiner, J. M., and Jansen, B., "Suppression of Pressure Loads in Cavity Flows," AIAA Journal, Vol. 42, No. 1, 2004, pp. 70-79. doi: $10.2514 / 1.9032$

[11] Bastrzyk, M., and Raman, G., "Cavity Noise Suppression Through Shear Layer Liftoff," 15th AIAA/CEAS Aeroacoustics Conference, AIAA Paper 2009-3203, May 2009. doi: $10.2514 / 6.2009-3203$

[12] Hsu, J., and Ahuja, K., "Cavity Noise Control Using Helmholtz Resonators," AIAA Paper 1996-1675, May 1996. doi:10.2514/6.1996-1675

[13] Cattafesta, L., Williams, D., Rowley, C., and Alvi, F., "Review of Active Control of Flow-Induced Cavity Oscillations," AIAA Paper 2003-3567, June 2003 doi: $10.2514 / 6.2003-3567$
[14] Rowley, C. W., and Williams, D. R., "Dynamics and Control of High-Reynolds-Number Flow over Open Cavities," Annual Review of Fluid Mechanics, Vol. 38, No. 1, 2006, pp. 251-276. doi:10.1146/annurev.fluid.38.050304.092057

[15] Rockwell, D., and Naudascher, E., "Review-Self-Sustained Oscillations of Flow Past Cavities," Journal of Fluids Engineering, Vol. 100, No. 2, 1978, pp. 152-165. doi:10.1115/1.3448624

[16] Rossiter, J.E., "Wind Tunnel Experiments on the Flow over Rectangular Cavities at Subsonic and Transonic Speeds," Royal Aircraft Establishment TR 64037, No. 3438, RAE Farnborough, U.K., 1964.

[17] Yang, Y., Rockwell, D., Cody, K. L.-F., and Pollack, M., "Generation of Tones Due to Flow Past a Deep Cavity: Effect of Streamwise Length," Journal of Fluids and Structures, Vol. 25, No. 2, 2009, pp. 364-388. doi:10.1016/j.jfluidstructs.2008.05.003

[18] Oshkai, P., and Yan, T., "Experimental Investigation of Coaxial Side Branch Resonators," Journal of Fluids and Structures, Vol. 24, No. 4, 2008, pp. 589-603. doi:10.1016/j.jfluidstructs.2007.10.008

[19] Bennett, G. J., Verdugo, F. R., and Stephens, D. B., "Shear Layer Dynamics of a Cylindrical Cavity for Different Acoustic Resonance Modes," 15th International Symposium on Applications of Laser Techniques to Fluid Mechanics, Paper 1727, July 2010.

[20] Verdugo, F. R., Camussi, R., and Bennett, G. J., "Aeroacoustic Source Characterization Technique Applied to a Cylindrical Helmholtz Resonator," 18th International Congress on Sound and Vibration (ICSV18), The International Institute of Acoustics and Vibration, 2011, Paper 2062.

[21] Stephens, D. B., Verdugo, F. R., and Bennett, G. J., "Shear Layer Driven Acoustic Modes in a Cylindrical Cavity," Journal of Pressure Vessel Technology, Vol. 136, No. 5, 2014, Paper 051307. doi: $10.1115 / 1.4026866$

[22] Marsden, O., Bailly, C., Bogey, C., and Jondeau, E., "Investigation of Flow Features and Acoustic Radiation of a Round Cavity," Journal of Sound and Vibration, Vol. 331, No. 15, 2012, pp. 3521-3543. doi:10.1016/j.jsv.2012.03.017

[23] Kook, H., and Mongeau, L., "Analysis of the Periodic Pressure Fluctuations Induced by Flow over a Cavity," Journal of Sound and Vibration, Vol. 251, No. 5, 2002, pp. 823-846. doi:10.1006/jsvi.2001.4013

[24] Ma, R., Slaboch, P. E., and Morris, S. C., "Fluid Mechanics of the Flow-Excited Helmholtz Resonator," Journal of Fluid Mechanics, Vol. 623, March 2009, pp. 1-26. doi:10.1017/S0022112008003911

[25] Foster, A., Swain, M., Barrett, R., D'Agaro, P., and James, S., "Effectiveness and Optimum Jet Velocity for a Plane Jet Air Curtain Used to Restrict Cold Room Infiltration," International Journal of Refrigeration, Vol. 29, No. 5, 2006, pp. 692-699. doi:10.1016/j.ijrefrig.2005.12.011

[26] Hu, L., Zhou, J., Huo, R., Peng, W., and Wang, H., "Confinement of Fire-Induced Smoke and Carbon Monoxide Transportation by Air Curtain in Channels," Journal of Hazardous Materials, Vol. 156, No. 1, 2008, pp. 327-334. doi:10.1016/j.jhazmat.2007.12.041

[27] Guyonnaud, L., Solliec, C., de Virel, M. D., and Rey, C., "Design of Air Curtains Used for Area Confinement in Tunnels," Experiments in Fluids, Vol. 28, No. 4, 2000, pp. 377-384. doi:10.1007/s003480050397

[28] Felis, F., Pavageau, M., Elicer-Cortés, J., and Dassonville, T., "Simultaneous Measurements of Temperature and Velocity Fluctuations in a Double Stream-Twin Jet Air Curtain for Heat Confinement in Case of Tunnel Fire," International Communications in Heat and Mass Transfer, Vol. 37, No. 9, 2010, pp. 1191-1196. doi:10.1016/j.icheatmasstransfer.2010.07.018

[29] Wickerhoff, J., and Sijpkes, T., "Aeroplane Provided with NoiseReducing Means, as well as a Landing Gear and Blowing Means," U.S. Patent Application 10/621,362, July 2003.

[30] Oerlemans, S., and de , and Bruin, A., "Reduction of Landing Gear Noise Using an Air Curtain," 15th AIAA/CEAS Aeroacoustics Conference (30th AIAA Aeroacoustics Conference), AIAA Paper 20093156, May 2009. doi:10.2514/6.2009-3156

[31] Zhao, K., Okolo, P. N., Kennedy, J., and Bennett, G. J., "A Study of Planar Jet Flow Control and Perforated Fairings for the Reduction of the Flow-Induced Noise of Tandem Rods in a Cross-Flow," 22nd AIAA/ CEAS Aeroacoustics Conference, AIAA Paper 2016-2772, June 2016. doi:10.2514/6.2016-2772 
[32] Zhao, K., Yang, X.-X., Okolo, P., Zhang, W.-H., and Bennett, G., "Use of a Plane Jet for Flow-Induced Noise Reduction of Tandem Rods," Chinese Physics B, Vol. 25, No. 6, 2016, Paper 064301. doi:10.1088/1674-1056/25/6/064301

[33] Zhao, K., Yang, X., Okolo, P., Wu, Z., and Bennett, G., "Use of Dual Planar Jets for the Reduction of Flow-Induced Noise," AIP Advances, Vol. 7, No. 2, 2017, Paper 025312. doi:10.1063/1.4976336

[34] Zhao, K., Okolo, P., Kennedy, J., and Bennett, G., "2D PIV Measurement on the Interaction Zone Between Two Parallel Planar Jets in a Crossflow," AIP Advances, Vol. 7, No. 10, 2017, Paper 105104. doi:10.1063/1.5005017

[35] Ramaprian, B., and Haniu, H., "Turbulence Measurements in Plane Jets and Plumes in Crossflow," Univ. of Iowa, IIHR Rept. 266, Iowa City, IA, 1983.

[36] Zhao, K., "Investigation in the Use of Dual-Jet Air Curtains Applied to the Landing Gear Noise Reduction," Ph.D. Thesis, Univ. of Dublin, Dublin, Ireland, 2017.

[37] Zhao, K., Yang, X., Okolo, P., Wu, Z., Zhang, W., and Bennett, G., "A Novel Method for Defining the Leeward Edge of the Planar Jet in Crossflow," Journal of Applied Fluid Mechanics, Vol. 10, No. 5, 2017, pp. $1475-1486$. doi:10.18869/acadpub.jafm.73.242.27617

[38] Ma, R., Slaboch, P. E., and Morris, S. C., "Fluid Mechanics of the FlowExcited Helmholtz Resonator," Journal of Fluid Mechanics, Vol. 623, March 2009, pp. 1-26. doi:10.1017/S0022112008003911

[39] Rodriguez Verdugo, F., Guitton, A., Camussi, R., and Grottadaurea, M., "Experimental Investigation of a Cylindrical Cavity," 15th AIAA/CEAS Aeroacoustics Conference, AIAA Paper 2009-3207, May 2009. doi:10.2514/6.2009-3207

[40] Chatellier, L., Laumonier, J., and Gervais, Y., "Theoretical and Experimental Investigations of Low Mach Number Turbulent Cavity Flows," Experiments in Fluids, Vol. 36, No. 5, 2004, pp. 728-740. doi:10.1007/s00348-003-0752-4

[41] El Hassan, M., Keirsbulck, L., and Labraga, L., "Aero-Acoustic Oscillations Inside Large Deep Cavities at Low-Subsonic Speeds," Journal of Fluids Engineering, Vol. 131, No. 1, 2007, Paper 011204.

[42] Heller, H. H., and Bliss, D. B., "The Physical Mechanism of Flow Induced Pressure Fluctuations in Cavities and Concepts for Suppression," 2nd AIAA Aeroacoustics Conference, AIAA Paper 1975-491, March 1975. doi:10.2514/6.1975-491

[43] Howe, M. S., "Edge, Cavity and Aperture Tones at Very Low Mach Numbers," Journal of Fluid Mechanics, Vol. 330, Jan. 1997, pp. 61-84. doi:10.1017/S0022112096003606

[44] Bennett, G., Stephens, D., and Rodriguez Verdugo, F., "Resonant Mode Characterisation of a Cylindrical Helmholtz Cavity Excited by a Shear Layer," Journal of the Acoustical Society of America, Vol. 141, No. 1, 2017, pp. 7-18. doi:10.1121/1.4973212

[45] Caruthers, J. E., Engels, R. C., and Ravinprakash, G. K., "A Wave Expansion Computational Method for Discrete Frequency Acoustics Within Inhomogeneous Flows," 2nd AIAA/CEAS Aeroacoustic Conference, AIAA Paper 1996-1684, May 1996. doi:10.2514/6.1996-1684
[46] Ruiz, G., and Rice, H. J., "An Implementation of a Wave-Based Finite Difference Scheme for a 3-D Acoustic Problem," Journal of Sound and Vibration, Vol. 256, No. 2, 2002, pp. 373-381. doi:10.1006/jsvi.2001.4218

[47] Bennett, G., O'Reilly, C., Liu, H., and Tapken, U., "Modelling MultiModal Sound Transmission from Point Sources in Ducts with Flow Using a Wave-Based Method," 16th International Congress on Sound and Vibration (ICSV16), The International Institute of Acoustics and Vibration, Kraków, Poland, 2009, pp. 4685-4693.

[48] Bliss, D., and Hayden, R., "Landing Gear and Cavity Noise Prediction," NASA-CR-2714, 1976.

[49] Neri, E., Kennedy, J., and Bennett, G., "Bay Cavity Noise for Full-Scale Nose Landing Gear: A Comparison Between Experimental and Numerical Results," Aerospace Science and Technology, Vol. 72, Jan. 2018, pp. 278-291. doi:10.1016/j.ast.2017.11.016

[50] Ben Khelil, S., Bardoux, P., Godard, J.-L., Garrec, T., Kennedy, J., and Bennett, G. J., "Investigation of the Noise Emission of a Regional Aircraft Main Landing Gear Bay," 23rd AIAA/CEAS Aeroacoustics Conference, AIAA Paper 2017-3012, June 2017. doi:10.2514/6.2017-3012

[51] Neri, E., Kennedy, J., and Bennett, G., "Reduction of Landing Gear Noise Using an Air Curtain," ASME 2015 Noise Control and Acoustics Division Conference at InterNoise 2015, ASME Paper NCAD20155911, 2015 doi:10.1115/NCAD2015-5911

[52] Kennedy, J., Neri, E., and Bennett, G., "The Reduction of Main Landing Gear Noise," 22nd AIAA/CEAS Aeroacoustics Conference, AIAA Paper 2016-2900, June 2016. doi:10.2514/6.2016-2900

[53] Verdugo, F. R., Guitton, A., and Camussi, R., "Experimental Investigation of a Cylindrical Cavity in a Low Mach Number Flow," Journal of Fluids and Structures, Vol. 28, Jan. 2012, pp. 1-19. doi:10.1016/j.jfluidstructs.2011.10.009

[54] Stephens, D. B., Rodriguez Verdugo, F., and Bennett, G. J., "Shear Layer Driven Acoustic Modes in a Cylindrical Cavity (AIAA 20103903)," 16th AIAA/CEAS Aeroacoustics Conference, AIAA Paper 2010-3903, June 2010. doi:10.2514/6.2010-3903

[55] Mendoza, J., and Ahuja, K., "Cavity Noise Control Through Upstream Mass Injection from a Coanda Surface," AIAA Paper 1996-1767, May 1996 doi:10.2514/6.1996-1767

[56] Zhao, K., Alimohammadi, S., Okolo, P. N., Kennedy, J., and Bennett, G. J., "Aerodynamic Noise Reduction Using Dual-Jet Planar Air Curtains," Journal of Sound and Vibration, Vol. 432, Oct. 2018, pp. 192-212. doi:10.1016/j.jsv.2018.06.036

[57] Zhao, K., Okolo, P. N., Wang, Y., Kennedy, J., and Bennett, G. J., "An Experimental Characterization of the Interaction Between Two Tandem Planar Jets in a Crossflow," Journal of Fluids Engineering, Vol. 140, No. 11, 2018, Paper 111106. doi: $10.1115 / 1.4040224$

D. Papamoschou Associate Editor 